\title{
The relation between the column density structures and the magnetic field orientation in the Vela $\mathrm{C}$ molecular complex
}

\author{
J. D. Soler ${ }^{1,2}$, P. A. R. Ade ${ }^{3}$, F. E. Angilè ${ }^{4}$, P. Ashton ${ }^{5}$, S. J. Benton ${ }^{6}$, M. J. Devlin ${ }^{4}$, B. Dober ${ }^{4,7}$, L. M. Fissel ${ }^{8}$,
} Y. Fukui ${ }^{9}$, N. Galitzki ${ }^{4}, 10$, N. N. Gandilo ${ }^{11}$, P. Hennebelle ${ }^{2}$, J. Klein ${ }^{4}$, Z.-Y. Li ${ }^{12}$, A. L. Korotkov ${ }^{13}$, P. G. Martin ${ }^{14}$, T. G. Matthews ${ }^{5}$, L. Moncelsi ${ }^{15}$, C. B. Netterfield ${ }^{16}$, G. Novak ${ }^{5}$, E. Pascale ${ }^{3}$, F. Poidevin ${ }^{17,18}$, F. P. Santos ${ }^{5}$, G. Savini ${ }^{19}$, D. $\operatorname{Scott}^{20}$, J. A. Shariff ${ }^{14}$, N. E. Thomas ${ }^{21}$, C. E. Tucker ${ }^{3}$, G. S. Tucker ${ }^{13}$, and D. Ward-Thompson ${ }^{22}$

${ }^{1}$ Max-Planck-Institute for Astronomy, Königstuhl 17, 69117 Heidelberg, Germany e-mail: soler@mpia.de

${ }^{2}$ Laboratoire AIM, Paris-Saclay, CEA/IRFU/SAp, CNRS, Université Paris Diderot, 91191 Gif-sur-Yvette Cedex, France

3 Cardiff University, School of Physics \& Astronomy, Cardiff, CF24 3AA, UK

4 Department of Physics \& Astronomy, University of Pennsylvania, Philadelphia, PA 19104, USA

5 Center for Interdisciplinary Exploration and Research in Astrophysics (CIERA) and Department of Physics \& Astronomy, Northwestern University, Evanston, IL 60208, USA

6 Department of Physics, Princeton University, Jadwin Hall, Princeton, NJ 08544, USA

7 National Institute of Standards and Technology (NIST), Boulder, CO 80305, USA

8 National Radio Astronomy Observatory (NRAO), Charlottesville, VA 22903, USA

9 Department of Physics and Astrophysics, Nagoya University, 464-8602 Nagoya, Japan

${ }^{10}$ Center for Astrophysics and Space Sciences, University of California, San Diego, CA 92093, USA

11 Johns Hopkins University, Baltimore, MD 21218, USA

12 Department of Astronomy, University of Virginia, Charlottesville, VA 22904, USA

13 Department of Physics, Brown University, Providence, RI 02912, USA

14 CITA, University of Toronto, Toronto, ON M5S 3H8, Canada

15 California Institute of Technology, Pasadena, CA 91125, USA

16 Department of Physics, University of Toronto, Toronto, ON M5S 1A7, Canada

17 Instituto de Astrofísica de Canarias, 38205 La Laguna, Tenerife, Spain

18 Universidad de La Laguna, Dpto. Astrofísica, 38206 La Laguna, Tenerife, Spain

19 Department of Physics \& Astronomy, University College London, London, WC1E 6BT, UK

20 Department of Physics \& Astronomy, University of British Columbia, Vancouver, BC V6T 1Z1, Canada

21 NASA/Goddard Space Flight Center, Greenbelt, MD 20771, USA

22 Jeremiah Horrocks Institute, University of Central Lancashire, PR1 2HE, UK

Received 13 February 2017 / Accepted 26 April 2017

\begin{abstract}
We statistically evaluated the relative orientation between gas column density structures, inferred from Herschel submillimetre observations, and the magnetic field projected on the plane of sky, inferred from polarized thermal emission of Galactic dust observed by the Balloon-borne Large-Aperture Submillimetre Telescope for Polarimetry (BLASTPol) at 250, 350, and 500 $\mu \mathrm{m}$, towards the Vela C molecular complex. First, we find very good agreement between the polarization orientations in the three wavelength-bands, suggesting that, at the considered common angular resolution of 3.0 that corresponds to a physical scale of approximately $0.61 \mathrm{pc}$, the inferred magnetic field orientation is not significantly affected by temperature or dust grain alignment effects. Second, we find that the relative orientation between gas column density structures and the magnetic field changes progressively with increasing gas column density, from mostly parallel or having no preferred orientation at low column densities to mostly perpendicular at the highest column densities. This observation is in agreement with previous studies by the Planck collaboration towards more nearby molecular clouds. Finally, we find a correspondence between (a) the trends in relative orientation between the column density structures and the projected magnetic field; and (b) the shape of the column density probability distribution functions (PDFs). In the sub-regions of Vela C dominated by one clear filamentary structure, or "ridges", where the high-column density tails of the PDFs are flatter, we find a sharp transition from preferentially parallel or having no preferred relative orientation at low column densities to preferentially perpendicular at highest column densities. In the sub-regions of Vela $\mathrm{C}$ dominated by several filamentary structures with multiple orientations, or "nests", where the maximum values of the column density are smaller than in the ridge-like sub-regions and the high-column density tails of the PDFs are steeper, such a transition is also present, but it is clearly less sharp than in the ridge-like sub-regions. Both of these results suggest that the magnetic field is dynamically important for the formation of density structures in this region.
\end{abstract}

Key words. astroparticle physics - dust, extinction - ISM: magnetic fields - ISM: clouds - submillimeter: ISM

\section{Introduction}

Magnetic fields are believed to play an important role in the formation of density structures in molecular clouds (MCs), from filaments to cores and eventually to stars (Crutcher 2012; Heiles \& Haverkorn 2012). However, their particular role in the general picture of MC dynamics is still controversial, mostly due to the lack of direct observations. 
A crucial tool for the study of the interstellar magnetic field is the observation of aligned dust grains, either via the polarization of background stars seen through MCs, or via maps of the polarization of the far-infrared or submillimetre emission from dust in the cloud (Hiltner 1949; Hildebrand 1988; Planck Collaboration Int. XIX 2015). Aspherical spinning dust particles preferentially align their rotation axis with the local direction of the magnetic field, producing linearly polarized emission that is perpendicular to the magnetic field (Davis \& Greenstein 1951; Lazarian 2000; Andersson et al. 2015). Thus, observations of the linear polarization provide an estimate of the magnetic field orientation projected on the plane of the sky and integrated along the line of sight, $\left\langle\hat{\boldsymbol{B}}_{\perp}\right\rangle$.

Recent observations by the Planck satellite (Planck Collaboration I 2016) have produced the first all-sky map of the polarized emission from dust at submillimetre wavelengths, providing an unprecedented data set in terms of sensitivity, sky coverage, and statistical significance for the study of $\left\langle\hat{\boldsymbol{B}}_{\perp}\right\rangle$. Over most of the sky, Planck Collaboration Int. XXXII (2016) analysed the relative orientation between column density structures and $\left\langle\hat{\boldsymbol{B}}_{\perp}\right\rangle$, inferred from the Planck $353-\mathrm{GHz}(850-\mu \mathrm{m})$ polarization observations at $15^{\prime}$ resolution, revealing that most of the elongated structures (filaments or ridges) are predominantly parallel to the $\left\langle\hat{\boldsymbol{B}}_{\perp}\right\rangle$ orientation measured on the structures. This statistical trend becomes less striking for increasing column density.

Within ten nearby $(d<450 \mathrm{pc})$ Gould Belt MCs, Planck Collaboration Int. XXXV (2016) measured the relative orientation between the total column density structures, $N_{\mathrm{H}}$ inferred from the Planck dust emission observations, and $\left\langle\hat{\boldsymbol{B}}_{\perp}\right\rangle$, inferred from the Planck $353-\mathrm{GHz}(850-\mu \mathrm{m})$ polarization observations at $10^{\prime}$ resolution. They showed that the relative orientation between $N_{\mathrm{H}}$ and $\left\langle\hat{\boldsymbol{B}}_{\perp}\right\rangle$ changes progressively with increasing $N_{\mathrm{H}}$, from preferentially parallel or having no preferred orientation at low $N_{\mathrm{H}}$ to preferentially perpendicular at the highest $N_{\mathrm{H}}$.

The results presented in Planck Collaboration Int. XXXV (2016) correspond to a systematic analysis of the trends described in previous studies of the relative orientation between $N_{\mathrm{H}}$ structures and $\left\langle\hat{\boldsymbol{B}}_{\perp}\right\rangle$ inferred from starlight polarization (Palmeirim et al. 2013; Li et al. 2013; Sugitani et al. 2011), as confirmed by the close agreement between the $\left\langle\hat{\boldsymbol{B}}_{\perp}\right\rangle$ orientations inferred from the Planck $353 \mathrm{GHz}$ polarization and starlight polarization observations presented in Soler et al. (2016). Subsequent studies of the relative orientation between $N_{\mathrm{H}}$ structures and $\left\langle\hat{\boldsymbol{B}}_{\perp}\right\rangle$ have identified similar trends to those described in Planck Collaboration Int. XXXV (2016), using $N_{\mathrm{H}}$ structures derived from Herschel observations at $20^{\prime \prime}$ resolution and $\left\langle\hat{\boldsymbol{B}}_{\perp}\right\rangle$ inferred from the Planck $353 \mathrm{GHz}$ polarization observations towards the high-latitude cloud L1642 (Malinen et al. 2016) as well as using $N_{\mathrm{H}}$ structures derived from Herschel observations together with starlight polarization (Cox et al. 2016).

The physical conditions responsible for the observed change in relative orientation between $N_{\mathrm{H}}$ structures and $\left\langle\hat{\boldsymbol{B}}_{\perp}\right\rangle$ are related to the degree of magnetization of the cloud (Hennebelle 2013; Soler et al. 2013). Soler et al. (2013) identified similar trends in relative orientation in simulations of molecular clouds where the magnetic field is at least in equipartition with turbulence, that is, trans- or sub-Alfvénic turbulence. This numerical interpretation, which has been further studied in Chen et al. (2016), is in agreement with the classical picture of MC formation, where the molecular cloud forms following compression of background gas, by the passage of the Galactic spiral shock or by an expanding supernova shell, and the compressed gas cools and so flows down the magnetic field lines to form a self-gravitating mass (Mestel 1965; Mestel \& Paris 1984).

In this paper, we extend the study of the relative orientation between $N_{\mathrm{H}}$ structures and $\left\langle\hat{\boldsymbol{B}}_{\perp}\right\rangle$ by using observations of the Vela $\mathrm{C}$ molecular complex obtained during the $2012 \mathrm{flight}$ of the Balloon-borne Large-Aperture Submillimetre Telescope for Polarimetry, BLASTPol (Pascale et al. 2012; Galitzki et al. 2014). Towards Vela C, BLASTPol provides unprecedented observations of the dust polarized emission in three different wavelength-bands (namely, 250, 350, and $500 \mu \mathrm{m}$ ) at 2.5 resolution, thus sampling spatial scales comparable to those considered in Planck Collaboration Int. XXXV (2016), but for a more distant, more massive, and more active MC.

Previous studies by the BLASTPol collaboration include an investigation of the relation between the total gas column density $N_{\mathrm{H}}$, the fractional polarization $p$, and the dispersion of orientation angles observed at $500 \mu \mathrm{m}$ towards the Vela $\mathrm{C}$ molecular complex, presented in Fissel et al. (2016). Also using the BLASTPol data, Gandilo et al. (2016) presented a study of the variation of $p$ in the three observed wavelength-bands towards the Vela $\mathrm{C}$ region, concluding that the polarization spectrum is relatively flat and does not exhibit a pronounced minimum at $\lambda \approx 350 \mu \mathrm{m}$, as suggested by previous measurements towards other MCs. Additionally, Santos et al. (2017) presented a quantitive comparison between the near-infrared (near-IR) polarization data from background starlight and the BLASTPol observations towards Vela C. In this new paper, we consider for the first time the analysis of the $\left\langle\hat{\boldsymbol{B}}_{\perp}\right\rangle$ orientations derived from the BLASTPol observations towards Vela $\mathbf{C}$.

This paper is organized as follows. In Sect. 2, we present the previously observed characteristics of Vela C. In Sect. 3, we introduce the BLASTPol polarization observations and the Herschel-based estimates of total gas column density. In Sect. 4, we introduce the method of using the histogram of relative orientations for quantifying the relation between $N_{\mathrm{H}}$ structures and $\left\langle\hat{\boldsymbol{B}}_{\perp}\right\rangle$. In Sect. 5, we discuss the results of our analysis. Section 6 gives our conclusions and anticipates future work. We reserve some additional analyses to three appendices. Appendix A presents the $\mathrm{HRO}$ analysis of Vela $\mathrm{C}$ based only on the BLASTPol observations. Appendix B presents a study of the results of the HRO analysis in the BLASTPol maps obtained with the different diffuse emission subtraction methods introduced in Fissel et al. (2016). Finally, Appendix C presents the HRO analysis of a portion of the Aquila rift based on the $N_{\mathrm{H}}$ values estimated from the Herschel observations and $\left\langle\hat{\boldsymbol{B}}_{\perp}\right\rangle$ estimated from the Planck-353 GHz polarization observations.

\section{The Vela $\mathrm{C}$ region}

Figure 1 shows dust column density and magnetic field observations toward the Vela Molecular Ridge (VMR), a collection of molecular clouds lying in the Galactic plane at distances ranging from approximately $700 \mathrm{pc}$ to $2 \mathrm{kpc}$ (Murphy \& May 1991; Liseau et al. 1992). The total molecular mass of the VMR, including four distinct cloud components labelled as Vela A, B, C, and D, amounts to about $5 \times 10^{5} M_{\odot}$ of gas (May et al. 1988; Yamaguchi et al. 1999). Netterfield et al. (2009) presented observations of Vela C in dust emission at 250, 350, and $500 \mathrm{mi}-$ crons, obtained using the Balloon-borne Large Aperture Submillimetre Telescope (BLAST, Pascale et al. 2008). That work confirmed that there are large numbers of objects in the early stages of star formation scattered throughout Vela $\mathrm{C}$, along with 


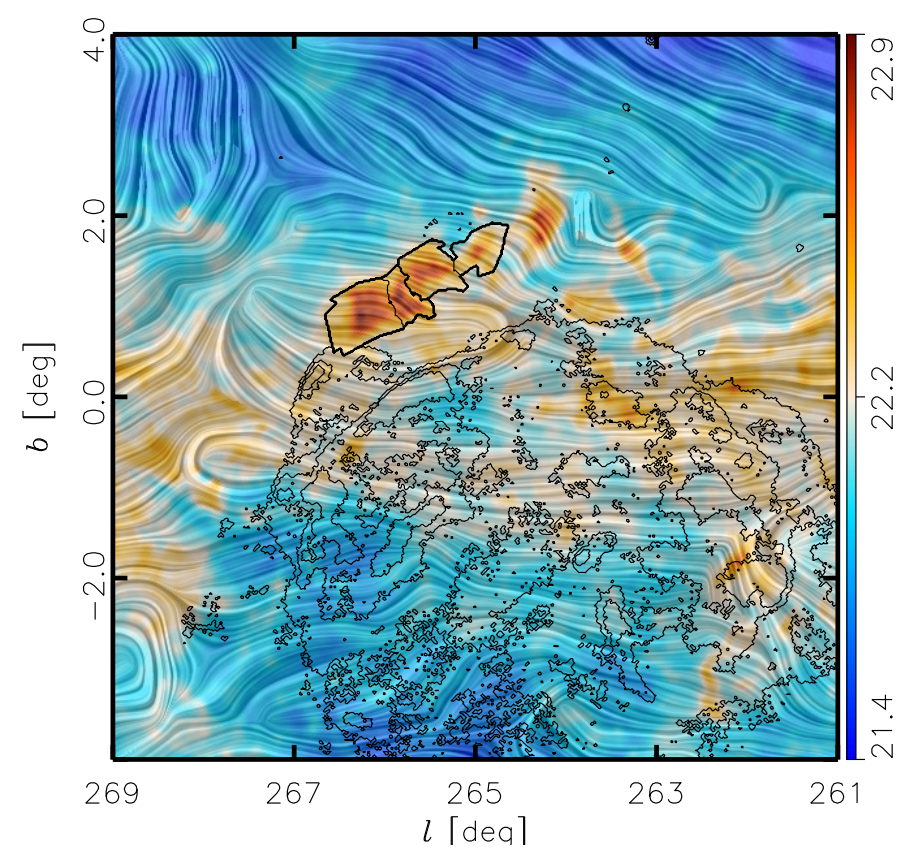

Fig. 1. Magnetic field and column density measured by Planck towards the region around the Vela $\mathrm{C}$ molecular complex. The colours represent the total column density, $N_{\mathrm{H}}$, inferred from the Planck observations (Planck Collaboration XI 2014). The "drapery" pattern, produced using the line integral convolution method (LIC, Cabral \& Leedom 1993), indicates the orientation of magnetic field lines, orthogonal to the orientation of the submillimetre polarization observed by Planck at $353 \mathrm{GHz}$. The thick black polygons correspond to the cloud sub-regions as defined in Hill et al. (2011). The narrow black lines represent the 12.7, 42.4 , and 84.9 counts per pixel contours in the X-ray energy band from 0.1 to $2.4 \mathrm{keV}$ observed with ROSAT(Aschenbach et al. 1995).

a well-known bright compact H II region, RCW 36 (Baba et al. 2004). Hill et al. (2011) mapped the dust emission from Vela $C$ using multiple Herschel wavelength bands, and identified five sub-regions with distinct characteristics which they named as North, Centre-Ridge, Centre-Nest, South-Ridge, and SouthNest. The last four of these were observed by BLAST-Pol and are indicated in Figs. 1 and 2.

Vela $\mathrm{C}$ has long been suspected to be a rare example of a nearby $(d \approx 700 \mathrm{pc}$; Liseau et al. 1992) and massive $(M \approx$ $10^{5} M_{\odot}$; Yamaguchi et al. 1999) molecular cloud at an early evolutionary stage. This conclusion stems from the observation that despite its relatively high mass, Vela $\mathrm{C}$ is characterized by extended regions of low temperature (Netterfield et al. 2009) and has produced only one or two late-type O-stars (in RCW 36; Baba et al. 2004).

\section{Observations}

In the present analysis we use two data sets. First, the Stokes $I$, $Q$, and $U$ observations obtained during the 2012 flight of BLASTPol. Second, the total column density maps derived from the Herschel satellite dust continuum observations.

\subsection{BLASTPol observations}

The balloon-borne submillimetre polarimeter BLASTPol and its Antarctic flights in 2010 and 2012, have been described

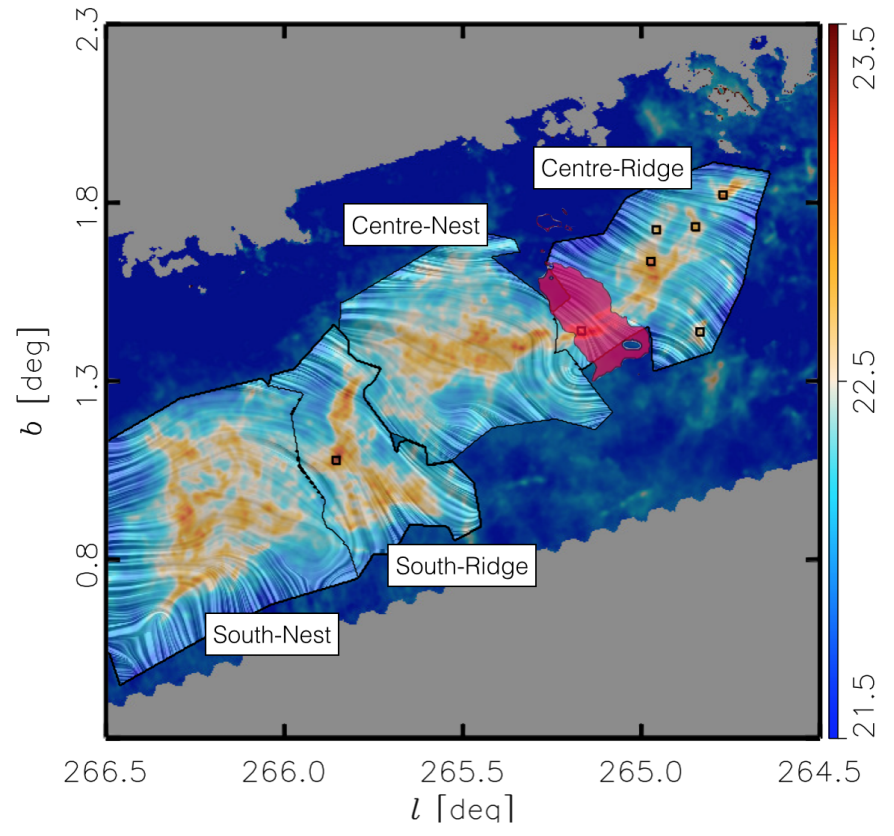

Fig. 2. Magnetic field and total intensity measured by BLASTPol towards the analysed sub-regions in the Vela $\mathrm{C}$ molecular complex. The colours represent $N_{\mathrm{H}}$, the total gas column density inferred from the Herschel observations. The "drapery" pattern, produced using the line integral convolution method (LIC, Cabral \& Leedom 1993), indicates the orientation of magnetic field lines, orthogonal to the orientation of the submillimetre polarization observed by BLASTPol at $500 \mu \mathrm{m}$. The black squares show the positions of the dense cores with $M>8 M_{\odot}$, the mass threshold considered in Hill et al. (2011), in the catalogue presented in Giannini et al. (2012). The large black polygons correspond to four of the five cloud sub-regions defined in Hill et al. (2011). The area in magenta corresponds to the region around RCW 36, where the dust temperature, derived from the Herschel observations, is larger than $20 \mathrm{~K}$.

by Pascale et al. (2012), Galitzki et al. (2014), Matthews et al. (2014), and Fissel et al. (2016). BLASTPol used a $1.8 \mathrm{~m}$ primary mirror to collect submillimetre radiation, splitting it into three wide wavelength bands $(\Delta \lambda / \lambda \approx 0.3)$ centred at 250,350 , and $500 \mu \mathrm{m}$. While the telescope scanned back and forth across the target cloud, the three wavelength bands were observed simultaneously by three detector arrays operating at $300 \mathrm{mK}$. The receiver optics included polarizing grids as well as an achromatic half-wave plate. The Vela $\mathrm{C}$ observations presented here were obtained as part of the 2012 Antarctic flight, during which the cloud was observed for $54 \mathrm{~h}$. The Stokes $I, Q$, and $U$ maps have already been presented by Fissel et al. (2016) and Gandilo et al. (2016).

Fissel et al. (2016) employed three methods for subtracting the contribution that the diffuse Galactic emission makes to the measured $I, Q$, and $U$ maps for Vela C; which referred to respectively as the "aggressive", "conservative", and "intermediate" methods. The aggressive method uses two reference regions located very close to the Vela $\mathrm{C}$ cloud (one on either side of it) to estimate the levels of polarized and unpolarized emission contributed by foreground and/or background dust unassociated with the cloud. These contributions are then removed from the measured $I, Q$, and $U$ maps. Because the reference regions are so close to the cloud, it is likely that they include some flux from material associated with Vela C. Thus, this method may over-correct, hence the name "aggressive". By contrast, the 
single reference region that is employed when the conservative method is used is more widely separated from Vela C, lying at a significantly higher Galactic latitude. This method may therefore under-correct. Finally, the intermediate diffuse emission subtraction method of Fissel et al. (2016) is the mean of the other two methods and was judged to be the most appropriate approach.

Naturally, the use of background subtraction imposes restrictions on the sky areas that may be expected to contain valid data following diffuse emission subtraction. Fissel et al. (2016) define a validity region outside of which the subtraction is shown to be invalid. With the exception of North and a very small portion of South-Ridge, all of the Hill et al. (2011) sub-regions are included in the validity region. Unless otherwise specified, we employed the intermediate diffuse emission subtraction approach. In Appendix B, we use the aggressive and conservative methods to quantify the extent to which uncertainties associated with diffuse emission subtraction affect our main results.

As noted by Fissel et al. (2016), the point spread function obtained by BLASTPol during our 2012 flight was several times larger than the prediction of our optics model. Furthermore, the beam was elongated. To obtain an approximately round beam, Fissel et al. (2016) smoothed their 500- $\mu$ m data to 2.5 FWHM resolution. Gandilo et al. (2016) alternatively smoothed all three bands to approximately 5.0 resolution in order to compare with Planck results for VelaC. For the purposes of this work, we require similarly shaped and nearly round beams at all three wavelengths, but we also do not want to sacrifice resolution. We were able to achieve these goals by smoothing all three bands to a resolution of 3.'0 FHWM.

\subsection{Column density maps}

The column density maps of Vela $\mathrm{C}$ were derived from the publicly available Herschel SPIRE and PACS data. SPIRE uses nearly identical filters to BLASTPol, but has higher spatial resolution (FWHM of 17.'6, 23.'9, and 35.'2 for the 250-, 350-, and $500-\mu \mathrm{m}$ bands, respectively). Data taken with the PACS instrument in a band centred at $160 \mu \mathrm{m}$ (FWHM of 13."6) were used to provide additional sensitivity to warm dust. These Herschelbased $N_{\mathrm{H}}$ maps were generated using Scanamorphos (Roussel 2013) and additional reduction and data manipulation was performed in the Herschel Interactive Processing Environment (HIPE version 11) including the Zero Point Correction function for the SPIRE maps. The resulting maps were smoothed to 35."2 resolution by convolving with Gaussian kernels of an appropriate size and then re-gridding to match the Herschel 500- $\mu$ m map.

We attempted to separate the Galactic foreground and background dust emission from the emission of Vela $\mathrm{C}$ following the procedure described in Sect. 5 of Fissel et al. (2016). Modified blackbody spectral energy distribution (SED) fits were made for each map pixel using the methods described in Hill et al. (2011) and with the dust opacity law presented in Hildebrand (1983) for a dust spectral index $\beta=2$. The result of these fits are column density $(N)$ and dust temperature $(T)$ maps, both at $35^{\prime \prime} 2$ resolution. It should be noted that above a temperature of approximately $20 \mathrm{~K}$, the dust emission is expected to peak at wavelengths shorter than $160 \mu \mathrm{m}$. For these warmest lines of sight (LOSs) our estimates will have a higher degree of uncertainty. Note also that we computed maps of the column density of atomic hydrogen, $N_{\mathrm{H}}$, while Hill et al. (2011) calculated the column density of molecular hydrogen, $N_{\mathrm{H}_{2}}$.

\section{Methods}

\subsection{The histogram of relative orientations}

We quantified the relative orientation between the iso- $N_{\mathrm{H}}$ contours and $\left\langle\hat{\boldsymbol{B}}_{\perp}\right\rangle$ using the histogram of relative orientations (HRO, Soler et al. 2013). In this technique, the $N_{\mathrm{H}}$ structures are characterized by their gradients, which are, by definition, perpendicular to the iso- $N_{\mathrm{H}}$ curves. The gradient constitutes a vector field that we can compare pixel by pixel to the $\left\langle\hat{\boldsymbol{B}}_{\perp}\right\rangle$ orientation inferred from the polarization maps.

We computed the angle $\phi$ between $\left\langle\hat{\boldsymbol{B}}_{\perp}\right\rangle$ and the tangent to the $N_{\mathrm{H}}$ contours,

$\phi_{\lambda}=\arctan \left(\left|\nabla N_{\mathrm{H}} \times \hat{\boldsymbol{E}}_{\lambda}\right|, \nabla N_{\mathrm{H}} \cdot \hat{\boldsymbol{E}}_{\lambda}\right)$.

For each observation band, characterized by its central wavelength $\lambda$, we assume that $\left\langle\hat{\boldsymbol{B}}_{\perp}\right\rangle$ is perpendicular to the unit polarization pseudo-vector $\hat{\boldsymbol{E}}_{\lambda}$. The orientation of $\hat{\boldsymbol{E}}_{\lambda}$ is defined by the polarization angle $\psi_{\lambda}$ calculated from the observed Stokes parameters using

$\psi_{\lambda}=\frac{1}{2} \arctan \left(-U_{\lambda}, Q_{\lambda}\right)$

In Eq. (1), as implemented, the norm carries a sign when the range used for $\phi_{\lambda}$ is between $0^{\circ}$ and $90^{\circ}$. For the sake of clarity in the representation of the HROs, we chose the range $0<\phi_{\lambda}<$ $90^{\circ}$, in contrast with the HROs presented in Soler et al. (2013) and Planck Collaboration Int. XXXV (2016), where $-90<\phi_{\lambda}<$ $90^{\circ}$. This selection does not imply any loss of generality, given that the relative orientation is independent of the reference vector, that is, $\phi_{\lambda}$ is equivalent to $-\phi_{\lambda}$.

\subsection{Construction of the histograms}

We constructed the HROs using the BLASTPol observations of polarization at 250,350 , and $500 \mu \mathrm{m}$ and the gradient of the total gas column density map, $N_{\mathrm{H}}$, estimated from the Herschel observations. The upper panels of Fig. 3 present the maps used in the construction of the HROs. For completeness, we present the HROs calculated from the BLASTPol polarization and the gradient of the intensity observed by BLASTPol in the $500-\mu \mathrm{m}$ band, $I_{500}$, in Appendix A.

The $N_{\mathrm{H}}$ HROs have the advantage of providing a more precise estimate of the total gas column density, but the disadvantage of being estimated with a different instrument and at a different angular resolution. The improvement in angular resolution provides a larger dynamic range for evaluating the HROs at different column densities.

We calculated the gradient of the $N_{\mathrm{H}}$ (or $I_{500}$ ) maps using the Gaussian derivatives method described in Soler et al. (2013). To guarantee adequate sampling of the derivates in each case, we applied a $5 \times 5$ derivative kernel computed over a grid with pixel size equal to one third of the beam FWHM in each observation; that is $\Delta l=\Delta b=0$ '.83 for the BLASTPol $I_{500}$ map (discussed in Appendix A) and $\Delta l=\Delta b=0.21$ for the Herschel $N_{\mathrm{H}}$ map.

We computed the relative orientation angle, $\phi_{\lambda}$, introduced in Eq. (1), in all the pixels where BLASTPol polarization observations in each band are available. We selected the polarization observations in terms of their polarized intensity $P_{\lambda} \equiv \sqrt{Q_{\lambda}^{2}+U_{\lambda}^{2}}$, such that we only consider $\phi_{\lambda}$ where the polarization signal-tonoise ratios $(\mathrm{S} / \mathrm{N}) P / \sigma_{P}>3$. These values of the polarization 

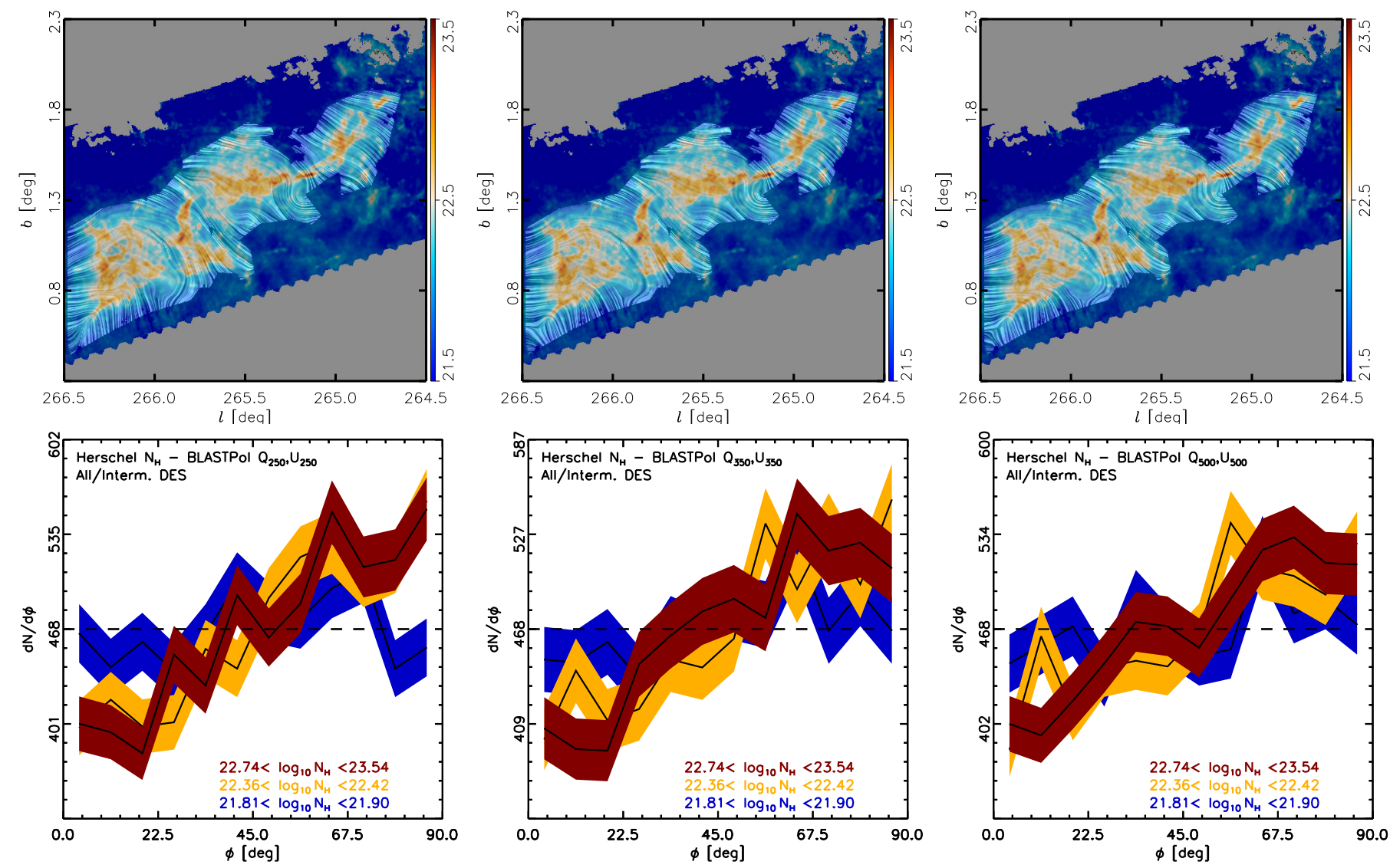

Fig. 3. Top: magnetic field and total intensity measured by BLASTPol towards two regions in the Vela molecular ridge. The colours represent $N_{\mathrm{H}}$, the total gas column density inferred from the Herschel observations. The "drapery" pattern, produced using the line integral convolution method (LIC, Cabral \& Leedom 1993), indicates the orientation of magnetic field lines, orthogonal to the orientation of the submillimetre polarization observed by BLASTPol at 250 (left), 350 (centre), and $500 \mu \mathrm{m}$ (right). Bottom: histogram of the relative orientations (HRO) between the iso- $N_{\mathrm{H}}$ contours and the magnetic field orientation inferred from the BLASTPol observations at 250 (left), 350 (centre), and $500 \mu \mathrm{m}$ (right). The figures present the HROs for the lowest bin, an intermediate bin, and the highest $N_{\mathrm{H}}$ bin (blue, orange, and dark red, respectively). These bins have equal numbers of selected pixels within the indicated $N_{\mathrm{H}}$ ranges. The horizontal dashed line corresponds to the average. The widths of the shaded areas for each histogram correspond to the $1 \sigma$ uncertainties related to the histogram binning operation. Histograms peaking at $0^{\circ}$ would correspond to $\left\langle\hat{\boldsymbol{B}}_{\perp}\right\rangle$ predominantly aligned with iso- $N_{\mathrm{H}}$ contours, while histograms peaking at $90^{\circ}$ would correspond to $\left\langle\hat{\boldsymbol{B}}_{\perp}\right\rangle$ predominantly perpendicular to iso- $N_{\mathrm{H}}$ contours.

$\mathrm{S} / \mathrm{N}$ correspond to classical uncertainties in the orientation angle $\sigma_{\psi}<9.5$ and it guarantees that the polarization bias is negligible (Serkowski 1958; Naghizadeh-Khouei \& Clarke 1993; Montier et al. 2015).

We computed the HROs in $15 N_{\mathrm{H}}$ bins, each with equal numbers of $\phi_{\lambda}$ values. This selection is intended to examine the change in $\phi_{\lambda}$ with increasing $N_{\mathrm{H}}$ with comparable statistics in each bin. The lower panels of Fig. 3 present the HROs for the lowest $N_{\mathrm{H}}$ bin, an intermediate $N_{\mathrm{H}}$ bin, and the highest $N_{\mathrm{H}}$ bin.

\subsection{Relative orientation parameter $\xi$}

The changes in the HROs are quantified using the histogram shape parameter $\xi$, defined as

$\xi=\frac{A_{0}-A_{90}}{A_{0}+A_{90}}$

where $A_{0}$ is the area under the histogram in the range $0^{\circ}<\phi<$ 22.5 and $A_{90}$ is the area under the histogram in the range $67.5<$ $\phi<90^{\circ} .0$. The value of $\xi$ is nearly independent of the number of bins selected to represent the histogram if the bin widths are smaller than the integration range.
A histogram peaking at $0^{\circ}$, corresponding to $\left\langle\hat{\boldsymbol{B}}_{\perp}\right\rangle$ mostly aligned with the $N_{\mathrm{H}}$ contours, would have $\xi>0$. A histogram peaking at $90^{\circ}$, corresponding to $\left\langle\hat{\boldsymbol{B}}_{\perp}\right\rangle$ mostly perpendicular to the $N_{\mathrm{H}}$ contours would have $\xi<0$. A flat histogram, corresponding to no preferred relative orientation between $\left\langle\hat{\boldsymbol{B}}_{\perp}\right\rangle$ and the $N_{\mathrm{H}}$ contours, would have $\xi \approx 0$.

The uncertainty in $\xi, \sigma_{\xi}$, is obtained from

$$
\sigma_{\xi}^{2}=\frac{4\left(A_{\mathrm{e}}^{2} \sigma_{A_{\mathrm{c}}}^{2}+A_{\mathrm{c}}^{2} \sigma_{A_{\mathrm{e}}}^{2}\right)}{\left(A_{\mathrm{c}}+A_{\mathrm{e}}\right)^{4}} .
$$

The variances of the areas, $\sigma_{A_{\mathrm{e}}}^{2}$ and $\sigma_{A_{\mathrm{c}}}^{2}$, characterize the "jitter" of the histograms. If the jitter is large, $\sigma_{\xi}$ is large compared to $|\xi|$ and the relative orientation is indeterminate. The jitter depends on the number of bins in the histogram, but $\xi$ does not.

We compared the $N_{\mathrm{H}}$ maps computed from the Herschel observations with the $\left\langle\hat{\boldsymbol{B}}_{\perp}\right\rangle$ estimates from the BLASTPol polarization observations. Given the distance to the cloud, this corresponds to comparing $N_{\mathrm{H}}$ structures on scales larger than $0.12 \mathrm{pc}$ to $\left\langle\hat{\boldsymbol{B}}_{\perp}\right\rangle$ on scales larger than $0.6 \mathrm{pc}$. This difference in scales implies that we are evaluating the relative orientation of $N_{\mathrm{H}}$ structures with respect to a larger-scale component of $\left\langle\hat{\boldsymbol{B}}_{\perp}\right\rangle$. 


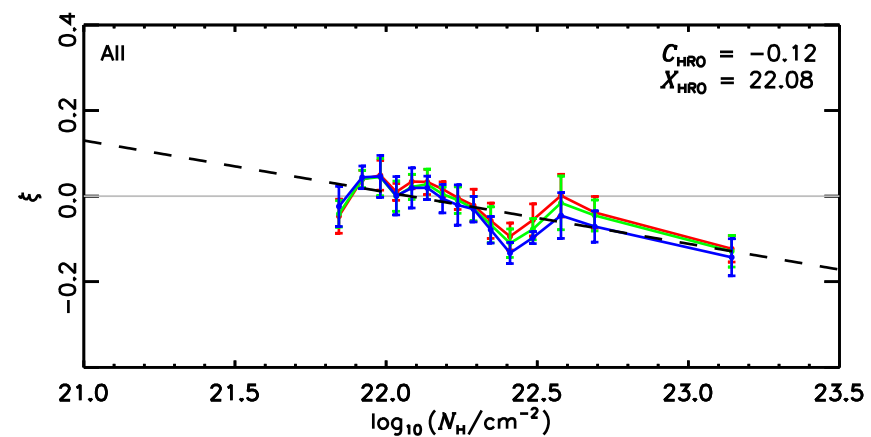

Fig. 4. Relative orientation parameter $\xi$, defined in Eq. (3), calculated for the different $N_{\mathrm{H}}$ bins towards the South-Nest, South-Ridge, CentreNest, and Centre-Ridge sub-regions of Vela C. The blue, green, and red curves correspond to the analysis of the BLASTPol observations at 250, 350 , and $500 \mu \mathrm{m}$, respectively. The values $\xi>0$ and $\xi<0$ correspond to the magnetic field being oriented mostly parallel or perpendicular to the iso- $N_{\mathrm{H}}$ contours, respectively. The black dashed line and the values of $C_{\mathrm{HRO}}$ and $X_{\mathrm{HRO}}$ correspond to the linear fit introduced in Eq. (5). The grey line is $\xi=0$, which corresponds to the case where there is no preferred relative orientation.

The $N_{\mathrm{H}}$ HROs, shown in the lower panels of Fig. 3, are flat in the lowest $N_{\mathrm{H}}$ range and peak at $90^{\circ}$ in the intermediate and highest $N_{\mathrm{H}}$ ranges across the three BLASTPol wavelength bands. The HROs corresponding to the highest and intermediate $N_{\mathrm{H}}$ ranges clearly show fewer counts for $0.0<\phi<22.5$ and more for $67.5<\phi<90.0$.

Figure 4 presents the behaviour of $\xi$ in different $N_{\mathrm{H}}$ bins and across the BLASTPol wavelength bands. The plot shows considerable agreement across the wavelength bands and shows that only in the highest $N_{\mathrm{H}}$ bin is there a clear indication of perpendicular orientation between $N_{\mathrm{H}}$ and $\left\langle\hat{\boldsymbol{B}}_{\perp}\right\rangle$, while $\xi$ for the rest of the $N_{\mathrm{H}}$ bins is consistent with no preferred relative orientation.

As in Planck Collaboration Int. XXXV (2016), we characterized the trends in relative orientation by assuming that the relation between $\xi$ and $\log _{10}\left(N_{\mathrm{H}} / \mathrm{cm}^{-2}\right)$ can be fit roughly by a linear relation

$\xi=C_{\mathrm{HRO}}\left[\log _{10}\left(N_{\mathrm{H}} / \mathrm{cm}^{-2}\right)-X_{\mathrm{HRO}}\right]$.

The measurements in each of the BLASTPol wavelength bands are in principle independent determinations of $\left\langle\hat{\boldsymbol{B}}_{\perp}\right\rangle$. Consequently, we used the estimates of $\xi$ in each wavelength band as independent points in the linear regression that we employed to estimate the values of $C_{\mathrm{HRO}}$ and $X_{\mathrm{HRO}}$.

\section{Discussion}

We observe that the relative orientation is consistent across the three wavelength bands observed in polarization by BLASTPol. This finding takes the results of Planck Collaboration Int. XXXV (2016), which were based exclusively on $353-\mathrm{GHz}(850-\mu \mathrm{m})$ polarization observations, and extends them not only to a different cloud, but also to the $\left\langle\hat{\boldsymbol{B}}_{\perp}\right\rangle$ morphology observed in three bands at higher frequencies. The interpretation of the agreement of the HRO analysis across these three wavelength bands is discussed in Sect. 5.1.

The results of the HRO analysis in Vela $\mathrm{C}$ suggest that, as in the MCs studied in Planck Collaboration Int. XXXV (2016), the magnetic field plays a significant role in the assembly of the parcels of gas that become MCs, as also suggested by the
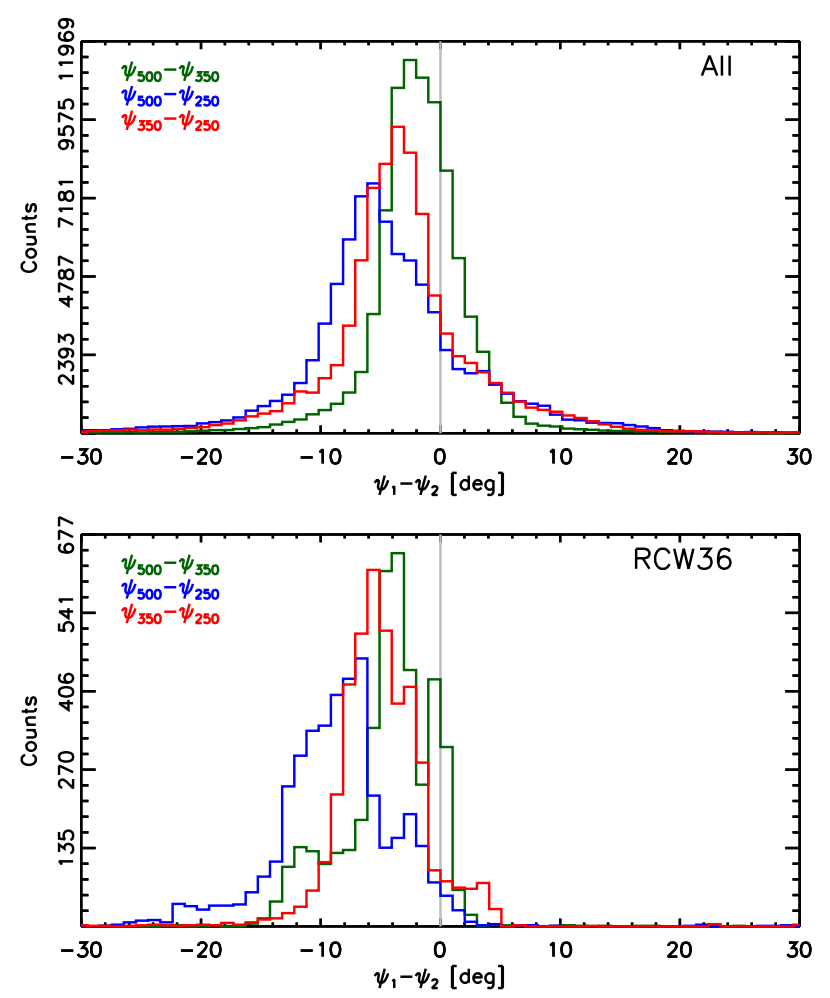

Fig. 5. Histogram of the polarization angle differences between the observations at the different BLASTPol wavelength bands towards Vela C sub-regions defined in Hill et al. (2011) (top) and in the vicinity of the RCW 36 region (bottom), that we have defined as the portion of the Centre-Ridge sub-region where the dust temperature derived from the Herschel observations is greater than $20 \mathrm{~K}$, as shown in Fig. 2.

analysis of simulations of MHD turbulence (Soler et al. 2013; Walch et al. 2015; Chen et al. 2016). We discuss this in Sect. 5.2 along with the possible relation between the relative orientation and the distributions of column density in different sub-regions of the Vela C clouds.

\subsection{Polarization angles at different wavelengths}

Figure 4 shows that the relative orientation between the $N_{\mathrm{H}}$ structures and $\left\langle\hat{\boldsymbol{B}}_{\perp}\right\rangle$ inferred from the three BLASTPol wavelength bands is very similar. This agreement is expected if we consider the small differences between the polarization angles in the different wavelength bands, which we compute directly and present in the top panel of Fig. 5. The average differences between orientation angles in different bands are all less than $7^{\circ}$, and hence have a negligible effect on the shape of the HROs and the behaviour of $\xi$ as function of $N_{\mathrm{H}}$.

The most widely accepted mechanism of dust grain alignment, that of radiative torques (RATs, Lazarian 2000), explains the changes in polarization properties across these bands as arising from the exposure of different regions to the interstellar radiation field (ISRF) and to internal radiation sources, such as RCW 36. According to RATs, dust grains in a cold region deep inside the cloud and far from internal radiation sources will not be as efficiently aligned as the dust grains in regions where they can be spun up by a more intense radiation field.

To investigate whether the polarization properties across the BLASTPol wavelengths depend on the environment in different regions of the clouds, Gandilo et al. (2016) evaluated the variations of the polarization fractions, $p_{\lambda}$, in different ranges of 
J. D. Soler et al.: Relative orientation between $\left\langle\hat{\boldsymbol{B}}_{\perp}\right\rangle$ and $N_{\mathrm{H}}$ structures towards Vela C

Table 1. Differences in polarization angles across BLASTPol wavelength bands in two $\log _{10}\left(N_{\mathrm{H}} / \mathrm{cm}^{-2}\right)$ ranges.

\begin{tabular}{|c|c|c|c|c|c|c|}
\hline \multirow[t]{2}{*}{ Region $^{a}$} & \multicolumn{3}{|c|}{$\log _{10}\left(N_{\mathrm{H}} / \mathrm{cm}^{-2}\right)<22.0$} & \multicolumn{3}{|c|}{$\log _{10}\left(N_{\mathrm{H}} / \mathrm{cm}^{-2}\right)>22.0$} \\
\hline & $\left\langle\Delta \psi_{500-350}\right\rangle^{b}$ & $\left\langle\Delta \psi_{500-250}\right\rangle$ & $\left\langle\Delta \psi_{350-250}\right\rangle$ & $\left\langle\Delta \psi_{500-350}\right\rangle$ & $\left\langle\Delta \psi_{500-350}\right\rangle$ & $\left\langle\Delta \psi_{500-350}\right\rangle$ \\
\hline All regions $\ldots$ & -0.7 & -0.0 & 0.8 & -1.5 & -3.9 & -2.5 \\
\hline South-Nest . . . . . & 0.3 & 6.9 & $6: 6$ & $-2: 1$ & -2.3 & -0.5 \\
\hline South-Ridge ...... & -0.1 & -3.2 & -3.0 & -0.3 & -4.4 & -4.0 \\
\hline Centre-Nest $\ldots \ldots$ & -0.2 & $-2: 1$ & -1.9 & -0.7 & $-4: 6$ & -3.9 \\
\hline Centre-Ridge $\ldots .$. & $-2: 4$ & -5.2 & -2.7 & $-2: 8$ & -5.0 & -2.1 \\
\hline
\end{tabular}

Notes. ${ }^{(a)}$ As defined in Hill et al. (2011) and illustrated in Fig. 2. ${ }^{(b)}\left\langle\Delta \psi_{500-350}\right\rangle \equiv\left\langle\psi_{500}-\psi_{350}\right\rangle$.

$N_{\mathrm{H}}$ and temperature, $T$, across the Vela $\mathrm{C}$ region. Gandilo et al. (2016) reported that no significant trends of $p_{\lambda}$ were found in different $N_{\mathrm{H}}$ ranges, and additionally, no trends over most of the $T$-ranges, except for a particular behaviour for the highest $T$ data coming from the vicinity of RCW 36.

In a similar way, we have evaluated the differences in polarization orientation angles between different BLASTPol wavelength bands in different $N_{\mathrm{H}}$ ranges in Vela C and summarize the results in Table 1, where, for the sake of simplicity, we present only two $N_{\mathrm{H}}$ ranges, namely $\log _{10}\left(N_{\mathrm{H}} / \mathrm{cm}^{-2}\right)<22.0$ and $\log _{10}\left(N_{\mathrm{H}} / \mathrm{cm}^{-2}\right)>22.0$, which are the most relevant for the change in relative orientation between $N_{\mathrm{H}}$ structures and $\left\langle\hat{\boldsymbol{B}}_{\perp}\right\rangle$. We observe that the differences in orientation angles between bands are all less than $7^{\circ}$, and although the differences change somewhat with increasing column density, their values do not significantly affect the HROs in the different $N_{\mathrm{H}}$ bins.

Towards the bipolar nebula around RCW 36 (Minier et al. 2013), the ionization by the H II region and the related increase in dust temperature can potentially introduce differences in the $\left\langle\hat{\boldsymbol{B}}_{\perp}\right\rangle$ orientation across the BLASTPol wavelength bands. To test this, we evaluated the difference between the $\left\langle\hat{\boldsymbol{B}}_{\perp}\right\rangle$ orientation inferred from the observations at different wavelengths in the region around RCW 36, where the dust temperature, derived from the Herschel observations, is larger than $20 \mathrm{~K}$. The results, presented in the bottom panel of Fig. 5, indicate that the mean differences in $\left\langle\hat{\boldsymbol{B}}_{\perp}\right\rangle$ for the different BLASTPol wavelength bands are not significantly different from those found in the rest of the cloud. This implies that the magnetic field, and the dust that dominates the observed $\left\langle\hat{\boldsymbol{B}}_{\perp}\right\rangle$ orientations, are approximately the same for the three wavelength bands. This is not unexpected, given that the observed $\left\langle\hat{\boldsymbol{B}}_{\perp}\right\rangle$ is the result of the integration of the magnetic field projection weighted by the dust emission, and most of the dust is in the bulk of the cloud, which is most likely unaffected by RCW 36 .

\subsection{Relative orientations in different portions of the cloud}

It is likely that different parts of Vela $\mathrm{C}$ are at different evolutionary stages; the regions dominated by several filamentary structures with multiple orientations ("nests") are less evolved, and regions dominated by one clear filamentary structure ("ridges") are in a more advanced state of evolution. To test this hypothesis, in terms of the relative orientation between the $N_{\mathrm{H}}$ structures and $\left\langle\hat{\boldsymbol{B}}_{\perp}\right\rangle$, we computed the HROs separately in four sub-regions of Vela C, namely South-Ridge, Centre-Ridge, South-Nest, and Centre-Nest, as identified in Hill et al. (2011) and illustrated in Fig. 2.

The maps of the South and Centre portions of Vela C are presented in the top panels of Figs. 6 and 7, respectively. The middle and bottom panels of Figs. 6 and 7 show the HROs that correspond to the ridge and nest sub-regions of the South and Centre portions of Vela C. The comparison of these HROs indicates that there is a clear difference in relative orientations at the lowest $N_{\mathrm{H}}$ and the highest $N_{\mathrm{H}}$ bins in all of the sub-regions except for the South-Nest.

In the Centre-Ridge and the South-Ridge, the HRO corresponding to the lowest $N_{\mathrm{H}}$ bin is flat or slightly peaks around $0^{\circ}$, in contrast with the HROs in the intermediate and highest $N_{\mathrm{H}}$ bins, which peak at $90^{\circ}$. In the Centre-Nest region, the HROs at the highest and intermediate $N_{\mathrm{H}}$ bins are relatively flat, but the HRO in the lowest $N_{\mathrm{H}}$ bin shows more counts in the $0<\phi_{\lambda}<22.5^{\circ}$ range than in $67.5<\phi_{\lambda}<90^{\circ}$, suggesting alignment between the lowest $N_{\mathrm{H}}$ contours and $\left\langle\hat{\boldsymbol{B}}_{\perp}\right\rangle$ in this sub-region. In the South-Nest region, the HROs present a large amount of jitter, which makes it difficult to identify any preferential relative orientation, but the histogram in the intermediate $N_{\mathrm{H}}$ bin clearly shows fewer counts in the $0<\phi_{\lambda}<22.5^{\circ}$ range than in $67.5<\phi_{\lambda}<90^{\circ}$, suggesting that the $N_{\mathrm{H}}$ contours are mostly perpendicular to $\left\langle\hat{\boldsymbol{B}}_{\perp}\right\rangle$ at the highest $N_{\mathrm{H}}$ in this sub-region. However, a more effective evaluation of the change in relative orientation between the iso- $N_{\mathrm{H}}$ contours and $\left\langle\hat{\boldsymbol{B}}_{\perp}\right\rangle$ is made in terms of the values of $\xi$, which we show in Fig. 8.

\subsubsection{Nests vs. ridges}

It is clear from the values of $\xi$ in Fig. 8 that the change in relative orientation, from mostly parallel or having no preferred orientation to mostly perpendicular with increasing $\log _{10}\left(N_{\mathrm{H}} / \mathrm{cm}^{-2}\right)$, is more evident in the ridge regions. There is some indication of a similar change in relative orientation in the nest regions, but there the values of $\xi$ are closer to zero at the high-column-density end.

In terms of the linear fit used to characterize the behaviour of $\xi$ as function of $\log _{10}\left(N_{\mathrm{H}} / \mathrm{cm}^{-2}\right)$, introduced in Eq. (5), all of the sub-regions present negative values of the slope, $C_{\mathrm{HRO}}$, suggesting the change from $\xi>0$ (or $N_{\mathrm{H}}$ contours and $\left\langle\hat{\boldsymbol{B}}_{\perp}\right\rangle$ mostly parallel) to $\xi<0$ (or $N_{\mathrm{H}}$ contours and $\left\langle\hat{\boldsymbol{B}}_{\perp}\right\rangle$ mostly perpendicular) with increasing $\log _{10}\left(N_{\mathrm{H}} / \mathrm{cm}^{-2}\right)$, although this transition is only completely clear in the observations towards the South-Ridge region, which also presents the most negative value of $C_{\mathrm{HRO}}$. The values of $C_{\mathrm{HRO}}$, also summarized in Table 2, are comparable to those reported in Planck Collaboration Int. XXXV (2016) for 10 nearby MCs, where the steepest slope was found towards IC 5146 and the Aquila rift, with $C_{\mathrm{HRO}}=-0.68$ and -0.60 respectively, and the shallowest towards the Corona Australis, with $C_{\mathrm{HRO}}=-0.11$ as shown in Table 2 of that reference.

The values of $X_{\mathrm{HRO}}$, which roughly correspond to the $\log _{10}\left(N_{\mathrm{H}} / \mathrm{cm}^{-2}\right)$ values where $\xi$ changes from positive to negative, or where the relative orientation between the $N_{\mathrm{H}}$ contours and $\left\langle\hat{\boldsymbol{B}}_{\perp}\right\rangle$ changes from mostly parallel to mostly 

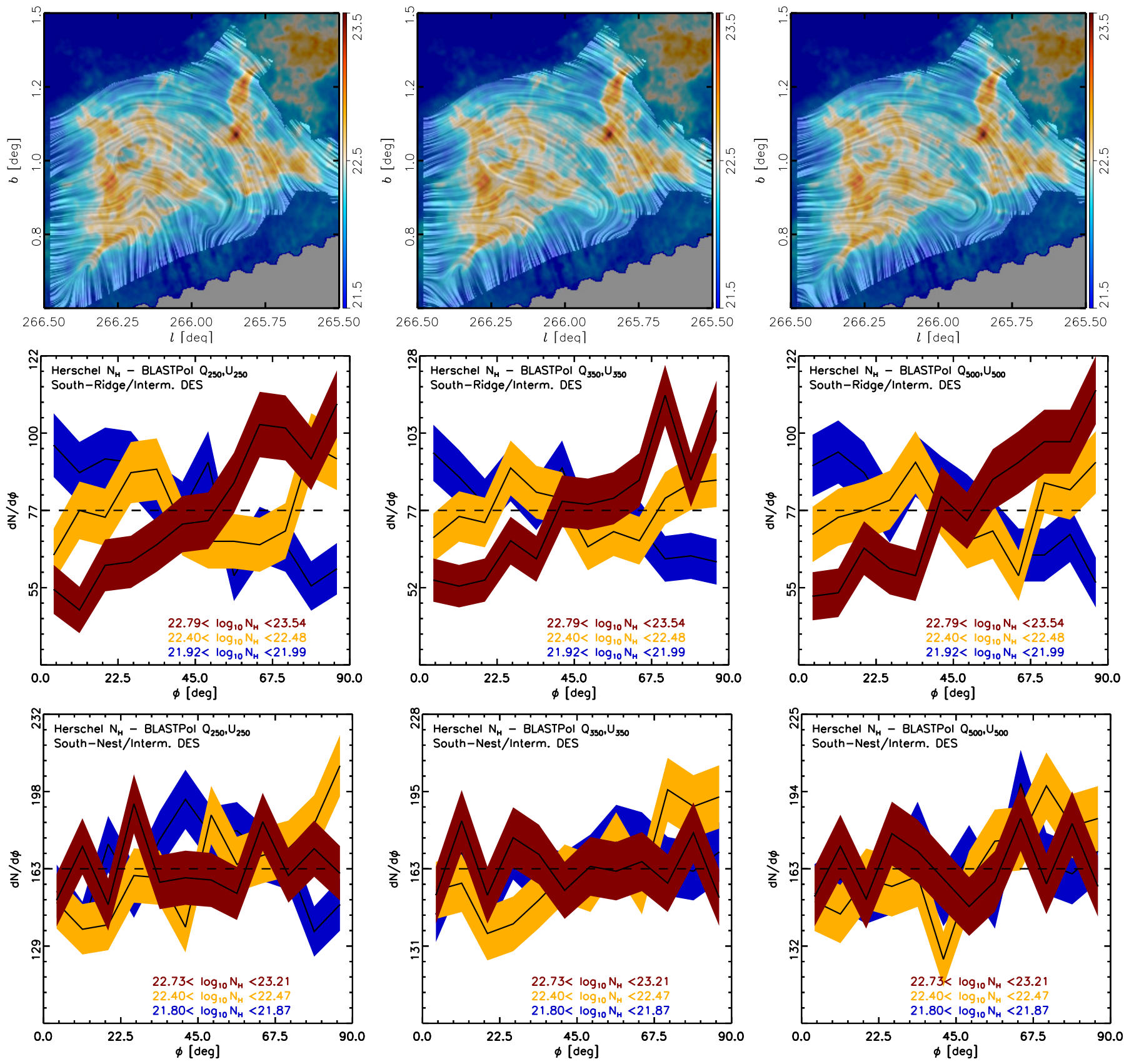

Fig. 6. Same as Fig. 3 for the South-Nest and South-Ridge regions of Vela C, as defined in Hill et al. (2011).

perpendicular, are also comparable with those in Planck Collaboration Int. XXXV (2016). The values of $X_{\text {HRO }}=22.71,22.39$, and 22.18 seen towards the Centre-Nest, the South-Ridge, and the South-Nest, respectively, are comparable to those found in Ophiuchus, $X_{\mathrm{HRO}}=22.70$, and the Aquila rift, $X_{\mathrm{HRO}}=22.23$. A particular case seems to be the Centre-Ridge, where $\xi$ is clearly less than zero for most $\log _{10}\left(N_{\mathrm{H}} / \mathrm{cm}^{-2}\right)$ values and oscillates around -0.2 for $\log _{10}\left(N_{\mathrm{H}} / \mathrm{cm}^{-2}\right)<21.7$, making the values of $X_{\mathrm{HRO}}$ and $C_{\mathrm{HRO}}$ not very informative. However, the mostly negative values of $\xi$ towards this regions are significant, given that the Centre-Ridge includes RCW 36 and, as commented by Hill et al. (2011), it contains most of the massive cores in Vela C.

When interpreting those results, it is possible that the different trends in the relative orientation between the $N_{\mathrm{H}}$ contours and $\left\langle\hat{\boldsymbol{B}}_{\perp}\right\rangle$ presented in Fig. 8 correspond to different projection effects in each one of the sub-regions, that is, the mean magnetic field has a different orientation with respect to the LOS, directly affecting the behaviour of $\xi$ as a function of $\log _{10}\left(N_{\mathrm{H}} / \mathrm{cm}^{-2}\right)$. From geometrical arguments, explained in detail in Appendix C of Planck Collaboration Int. XXXV (2016), it is unlikely that the relative orientation in the South-Ridge and Centre-Ridge is too far from the three-dimensional configuration of the magnetic field and the density structures. But it is possible that the values of $\xi$ around zero in the South-Nest and Centre-Nest are produced by the mean magnetic field being oriented close to the LOS, thus making it difficult to identify a preferential relative orientation between the projected density structure and $\left\langle\hat{\boldsymbol{B}}_{\perp}\right\rangle$. Alternatively, it is possible that the South-Nest and Centre-Nest are more turbulent, as suggested by the dispersion in $\left\langle\hat{\boldsymbol{B}}_{\perp}\right\rangle$ orientations inferred from the near-infrared polarimetry observations presented in Kusune et al. (2016). However, as described in the same reference, this hypothesis is not clearly justified by the width of 

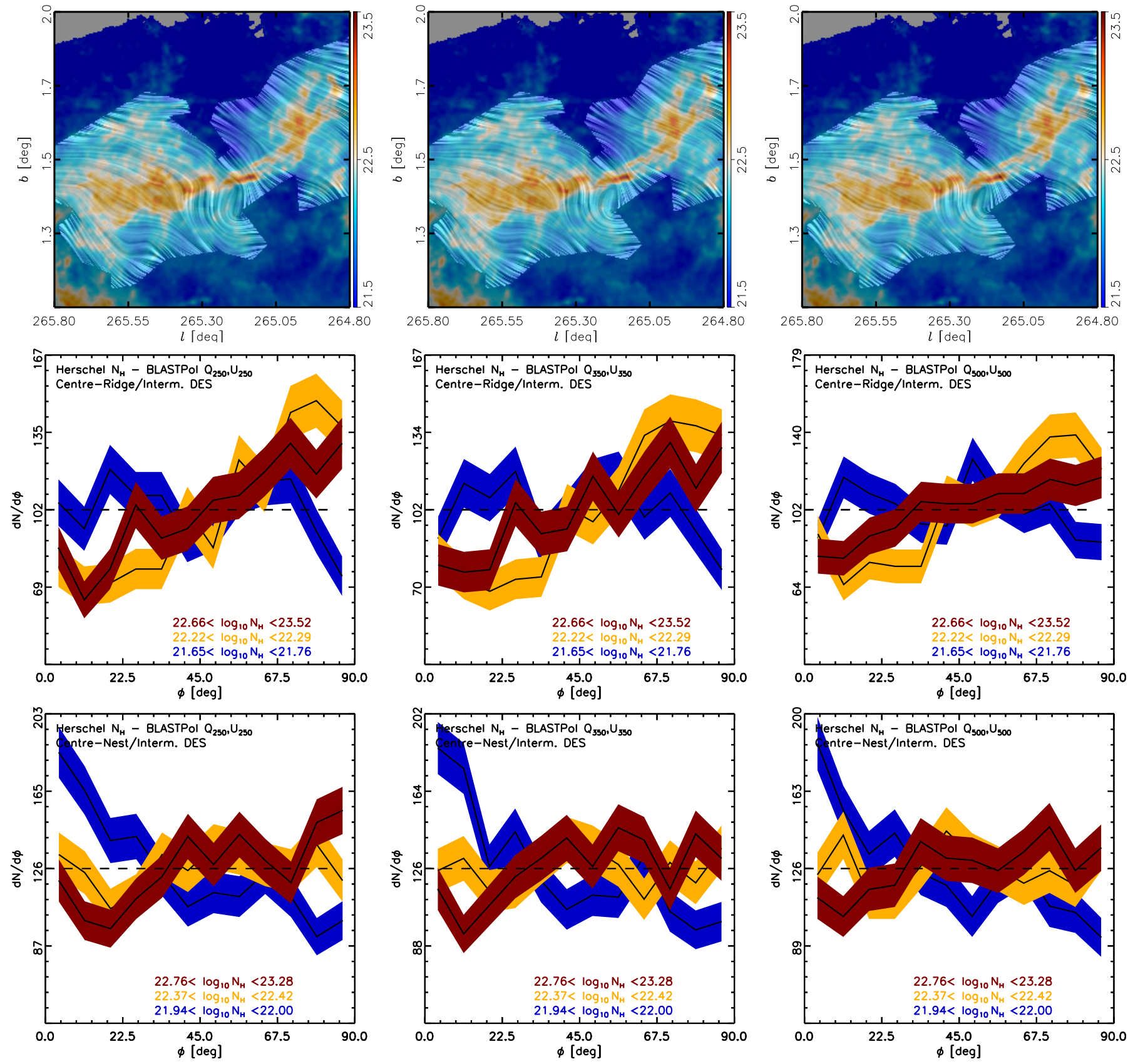

Fig. 7. Same as Fig. 3 for the Centre-Nest and Centre-Ridge regions of Vela C as defined in Hill et al. (2011).

${ }^{13} \mathrm{CO}$ emission lines, which are not correlated with the $\left\langle\hat{\boldsymbol{B}}_{\perp}\right\rangle$ dispersions in the sub-regions.

Detailed comparison between the $\left\langle\hat{\boldsymbol{B}}_{\perp}\right\rangle$ morphology and the velocity information inferred from different molecular tracers is beyond the scope of this work, but will be considered in a forthcoming publication (Fissel et al. 2017). For the moment, we simply compare the implications of the different relative orientations between $N_{\mathrm{H}}$ contours and $\left\langle\hat{\boldsymbol{B}}_{\perp}\right\rangle$ in the sub-regions of Vela $\mathrm{C}$, and consider this in relation to other cloud characteristics, such as the column density probability distribution functions.

\subsubsection{Column density probability distribution functions and relative orientations}

Hill et al. (2011) describe a significant difference between the ridge and nest sub-regions of Vela $C$ : while all the clouds have comparable masses, the highest values of the column density probability distribution functions (PDFs) are found in the ridge sub-regions, as illustrated in Fig. 9. Moreover, Hill et al. (2011) also indicate that most of the cores with $M>8 M_{\odot}$ are found in the Centre-Ridge region, while the Centre-Nest has two, the South-Ridge has one, and the South-Nest has none. This observation is confirmed by the distribution of dense cores in Vela C presented in Giannini et al. (2012), as illustrated in Fig. 2. Hill et al. (2011) interpret this as the effect of "constructive large-scale flows" and strong compression of material in the Centre-Ridge. The results of the HRO analysis indicate that such flows are parallel to the mean magnetic field direction, resulting in the high column density structure that is perpendicular to the magnetic field.

To test this hypothesis in a different region, we evaluated the column density PDFs and the distribution of massive cores 

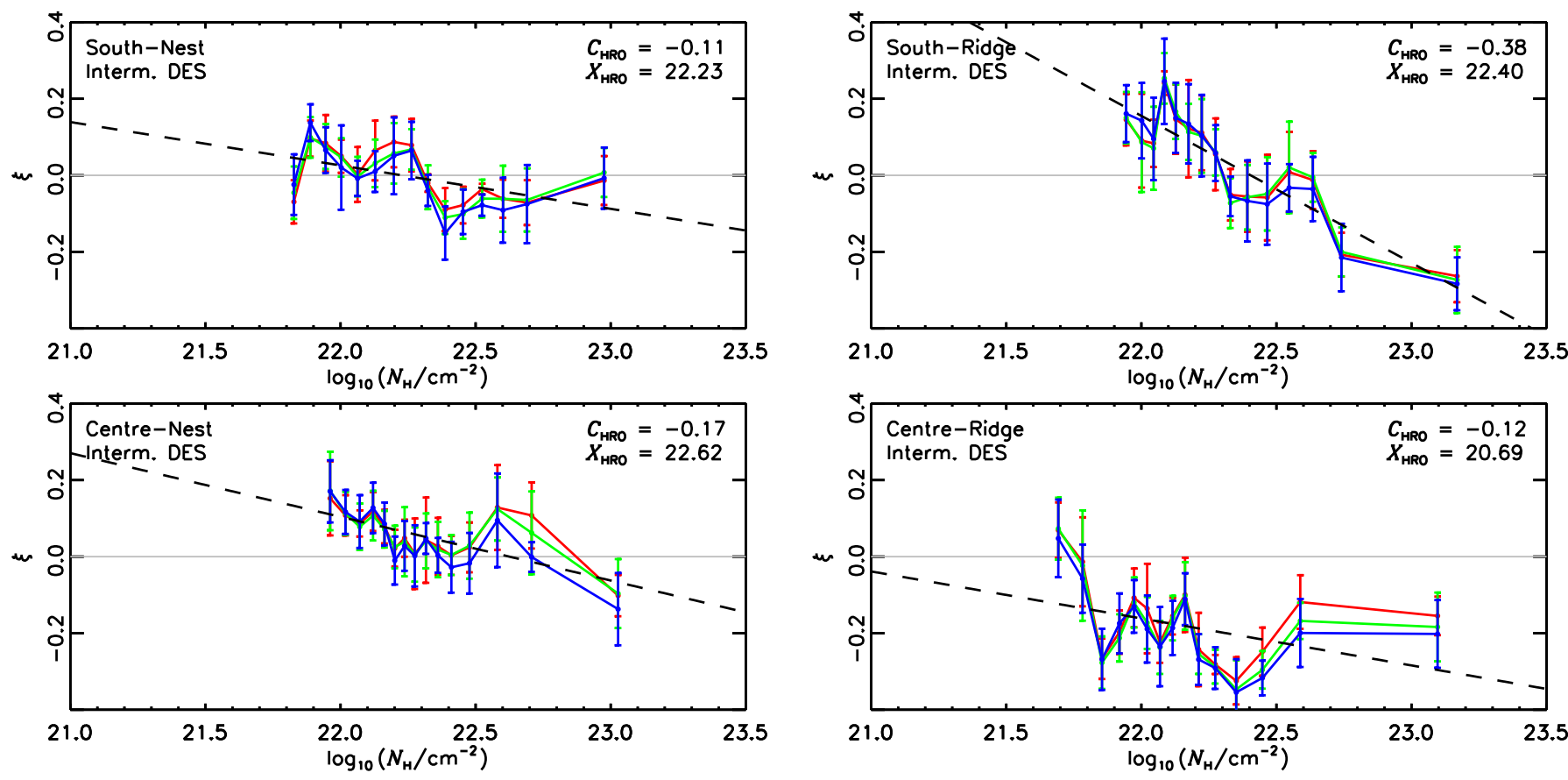

Fig. 8. Relative orientation parameter $\xi$, defined in Eq. (3), calculated for the different $N_{\mathrm{H}}$ bins towards the sub-regions of Vela $\mathrm{C}$ as defined in Hill et al. (2011) and illustrated in Fig. 2. The values $\xi>0$ and $\xi<0$ correspond to the magnetic field being oriented mostly parallel or perpendicular to the iso- $N_{\mathrm{H}}$ contours, respectively. The black dashed line and the values of $C_{\mathrm{HRO}}$ and $X_{\mathrm{HRO}}$ correspond to the linear fit introduced in Eq. (5). The grey line is $\xi=0$, which corresponds to the case where there is no preferred relative orientation.

and the relative orientation between $N_{\mathrm{H}}$ and $\left\langle\hat{\boldsymbol{B}}_{\perp}\right\rangle$ in the Serpens South sub-region of the Aquila complex, as described in detail in Appendix C. Serpens South is slightly less massive that Vela C, but it is less affected by feedback than Orion, which is the closest mass equivalent in the group of clouds studied in Planck Collaboration Int. XXXV (2016). The observations presented in Hill et al. (2012) indicate that the filamentary structure in the Vela C Centre-Ridge and the Serpens South filament are quite similar in terms of their column density profiles, and mass per unit length. Additionally, they are similar in that both contain an H II region, RCW 36 in the case of Vela C and W40 in the case of Serpens South.

The HRO analysis of Serpens South indicates that as in Vela $\mathrm{C}$, the maximum column densities, the flattest slopes of the column density PDF tail, and the largest number of dense cores are located in the portion of the cloud with the sharpest transition in the relative orientation between gas column density structures and the magnetic field. The transition is from $\left\langle\hat{\boldsymbol{B}}_{\perp}\right\rangle$ being mostly parallel or having no preferred alignment with respect to the $N_{\mathrm{H}}$ structures to mostly perpendicular; see Sect. 5.2.1 and Appendix C. This observational fact, which alone does not imply causality, is significant if we consider the current observations of the assembly of density structures in molecular clouds (André et al. 2014, and the references therein).

\subsection{The role of the magnetic field in molecular cloud formation}

Each of the sub-regions of Vela C contains roughly the same mass (Hill et al. 2011). Assuming a constant star-formation efficiency, each sub-region should therefore form approximately the same number of massive stars, which is not what is observed in terms of the column density PDF and high-mass core distribution. Then, the difference between the sub-regions may be

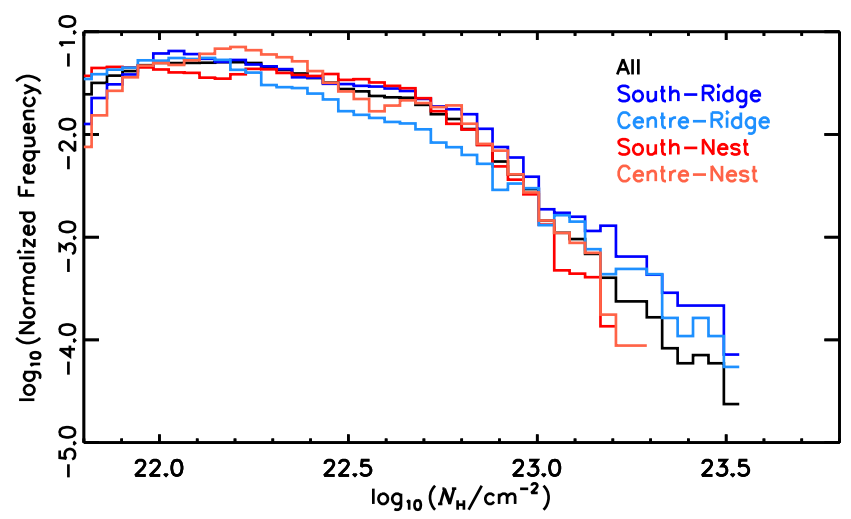

Fig. 9. Comparison of the normalized $N_{\mathrm{H}}$ probability distribution functions (PDFs), inferred from the Herschel observations towards the four sub-regions of Vela C presented in Fig. 2. The $y$-axis is presented in logarithmic scale for the sake of comparison with Fig. 5 of Hill et al. (2011).

in their efficiency for gathering material into the dense regions where star formation is ongoing.

Kirk et al. (2013) and Palmeirim et al. (2013) presented observational evidence of the potential feeding of material into hubs or ridges, in high- and low-mass star forming regions respectively, where the gathering flows seem to follow the magnetic field direction. The finding of ridge-like structures towards the Centre-Ridge and the South-Ridge, where the $N_{\mathrm{H}}$ structures are mostly perpendicular to $\left\langle\hat{\boldsymbol{B}}_{\perp}\right\rangle$, suggest that these sub-regions in Vela $C$ were formed through a similar mechanism.

Planck Collaboration Int. XXXV (2016) argue that the transition between mostly parallel and mostly perpendicular is related to the balance between the kinetic, gravitational, and magnetic energies and the flows of matter, which are restricted 
J. D. Soler et al.: Relative orientation between $\left\langle\hat{\boldsymbol{B}}_{\perp}\right\rangle$ and $N_{\mathrm{H}}$ structures towards Vela C

Table 2. Parameters of the relative orientation between $N_{\mathrm{H}}$ and $\left\langle\hat{\boldsymbol{B}}_{\perp}\right\rangle$ towards the Vela C sub-regions.

\begin{tabular}{ccccc}
\hline \hline Region & $C_{\mathrm{HRO}^{a}}$ & $X_{\mathrm{HRO}}{ }^{a}$ & $\operatorname{Max}\left(\log \left(N_{\mathrm{H}} / \mathrm{cm}^{-2}\right)\right)^{b}$ & $\operatorname{Mean}\left(\log \left(N_{\mathrm{H}} / \mathrm{cm}^{-2}\right)\right)^{b}$ \\
\hline South-Nest $\ldots \ldots$ & -0.11 & 22.23 & 22.9 & 22.1 \\
South-Ridge $\ldots \ldots$ & -0.38 & 22.40 & 23.3 & 22.2 \\
Centre-Nest $\ldots \ldots$ & -0.17 & 22.62 & 23.0 & 22.2 \\
Centre-Ridge $\ldots \ldots$ & -0.12 & 20.69 & 23.2 & 22.1 \\
\hline
\end{tabular}

Notes. ${ }^{(a)}$ Fit of $\xi$ vs. $\log _{10}\left(N_{\mathrm{H}} / \mathrm{cm}^{-2}\right)$. See Eq. (5) and Fig. 8. ${ }^{(b)}$ From Hill et al. (2011).

by the Lorentz force to follow the magnetic fields. A frozenin and strong interstellar magnetic field would naturally cause a self-gravitating, static cloud to become oblate, with its major axis perpendicular to the field lines, because gravitational collapse would be restricted to occurring along field lines (Mestel \& Spitzer 1956; Mestel \& Paris 1984; Mouschovias 1976).

In a dynamic picture of MCs, the matter-gathering flows, such as the ones driven by expanding bubbles (Inutsuka et al. 2015), are more successful at gathering material and eventually forming MCs if they are close to parallel to the magnetic field (Hennebelle \& Pérault 2000; Ntormousi et al. 2017). Less dense structures, which are not self-gravitating, would be stretched along the magnetic field lines by velocity shear, thereby producing aligned density structures, as discussed in Hennebelle (2013). Walch et al. (2015) report a similar effect for the magnetic field in a $500 \mathrm{pc}$ simulation of a Galactic disc including self-gravity, magnetic fields, heating and radiative cooling, and supernova feedback. In the evolution of their simulation setup, they observe that the magnetic field is delaying or favouring the collapse in certain regions by changing the amount of dense and cold gas formed. The additional magnetic pressure is significant in dense gas and thus slows the formation of dense and cold, molecular gas.

Given the aforementioned observational and theoretical considerations, it is plausible to consider that the Centre-Ridge is a more evolved region, where the structures produced by the flows along $\left\langle\hat{\boldsymbol{B}}_{\perp}\right\rangle$, which would be mostly parallel to $\left\langle\hat{\boldsymbol{B}}_{\perp}\right\rangle$, have already collapsed into the central object. This structure is gravitationally bound and collapsing into dense sub-structures, including high-mass stars, such as those found in RCW 36. This would imply that the South-Ridge is in an early state of accretion, where the column density structures that are mostly oriented along $\left\langle\hat{\boldsymbol{B}}_{\perp}\right\rangle$, at $\log _{10}\left(N_{\mathrm{H}} / \mathrm{cm}^{-2}\right)<22.6$, are the product of the inflows feeding the highest column density structures. In this scenario, the Center-Nest and South-Nest sub-regions would correspond to regions that are less efficient in gathering material due to interaction between matter-gathering flows and the magnetic field.

\section{Conclusions}

We have evaluated the relative orientations between the column density structure, $N_{\mathrm{H}}$, inferred from the Herschel satellite observations, and the magnetic field projected on the plane of sky, $\left\langle\hat{\boldsymbol{B}}_{\perp}\right\rangle$, inferred from observations of polarization at 250 , $350,500 \mu \mathrm{m}$ by BLASTPol, towards the Vela C molecular complex. We found that the relative orientation between the iso$N_{\mathrm{H}}$ contours and $\left\langle\hat{\boldsymbol{B}}_{\perp}\right\rangle$, changes progressively with increasing $N_{\mathrm{H}}$, from preferentially parallel or having no preferred orientation to preferentially perpendicular, in agreement with the behaviour reported by Planck Collaboration Int. XXXV (2016) in ten nearby MCs.

We found close agreement between the values of the $\left\langle\hat{\boldsymbol{B}}_{\perp}\right\rangle$ orientation inferred from observations in the three BLASTPol wavelength bands. Consistently, we found very similar trends in relative orientation between the iso- $N_{\mathrm{H}}$ contours and $\left\langle\hat{\boldsymbol{B}}_{\perp}\right\rangle$ in the three BLASTPol wavelength bands. This agreement, together with the flat polarization SED across the bands reported in Gandilo et al. (2016) suggest that the observed $\left\langle\hat{\boldsymbol{B}}_{\perp}\right\rangle$ orientation is dominated by regions in the bulk of the gas associated with the MC, which contain most of the dust and are exposed to a relatively homogeneous radiative environment that, at the considered scales, is less likely to produce significant changes in the dust grain alignment.

We studied the relative orientations between the iso- $N_{\mathrm{H}}$ contours and $\left\langle\hat{\boldsymbol{B}}_{\perp}\right\rangle$ towards different sub-regions of Vela $\mathrm{C}$. We found different behaviours in the sub-regions dominated by a single elongated structure, or "ridge", and the sub-regions dominated by multiple filamentary structures, or "nests". Towards the Centre-Ridge, where RCW 36, the highest maximum values of $N_{\mathrm{H}}$, the shallowest $N_{\mathrm{H}}$-PDF power-law tail, and most of the massive cores are found, we see that the iso- $N_{\mathrm{H}}$ contours are mostly perpendicular to $\left\langle\hat{\boldsymbol{B}}_{\perp}\right\rangle$. Towards the South Ridge sub-region, where the slope of the $N_{\mathrm{H}}$-PDF power-law tail is comparable to the Centre-Ridge sub-region, we see a steep transition from $N_{\mathrm{H}}$ structures and $\left\langle\hat{\boldsymbol{B}}_{\perp}\right\rangle$ being mostly parallel at $\log _{10}\left(N_{\mathrm{H}} / \mathrm{cm}^{-2}\right)<22.4$ to mostly perpendicular at $\log _{10}\left(N_{\mathrm{H}} / \mathrm{cm}^{-2}\right)>22.4$. In contrast, towards the Centre-Nest and the South-Nest, where the power-law tails of the $N_{\mathrm{H}}-\mathrm{PDF}$ are steeper and the maximum $N_{\mathrm{H}}$ values are lower than in the ridgelike regions, we see a less steep transition from $N_{\mathrm{H}}$ structures and $\left\langle\hat{\boldsymbol{B}}_{\perp}\right\rangle$ being mostly parallel at low $N_{\mathrm{H}}$ to mostly perpendicular at the highest $N_{\mathrm{H}}$. We found a similar behaviour towards the Serpens South region in Aquila, where we compared the column density structure inferred from the Herschel satellite observations and $\left\langle\hat{\boldsymbol{B}}_{\perp}\right\rangle$ inferred from observations of polarization by Planck at $353 \mathrm{GHz}(850 \mu \mathrm{m})$. These observational results suggest that the magnetic field is important in gathering the gas that composes the molecular cloud, and a remnant of that assembly process is present in the relative orientation of the projected density and magnetic field.

Future studies of the molecular emission towards Vela C would enable the understanding of the kinematics in this region, which would help determine how the ridge-like and the nest-like sub-regions came to be. Further comparison of the relative orientation between the $N_{\mathrm{H}}$ structures and $\left\langle\hat{\boldsymbol{B}}_{\perp}\right\rangle$ with the population of cores in other molecular clouds would also help us understand how the magnetic field influences the formation of density structures, and potentially how the cloud-scale magnetic environment is related to the formation of stars. 
Acknowledgements. The BLASTPol collaboration acknowledges support from NASA through grant numbers NAG5-12785, NAG5-13301, NNGO-6GI11G, NNX0-9AB98G, and the Illinois Space Grant Consortium, the Canadian Space Agency, the Leverhulme Trust through the Research Project Grant F/00 407/BN, Canada's Natural Sciences and Engineering Research Council, the Canada Foundation for Innovation, the Ontario Innovation Trust, and the US National Science Foundation Office of Polar Programs. This work was possible through the funding from the European Research Council under the European Community's Seventh Framework Programme (FP7/2007-2013 Grant Agreement Nos. 306483 and 291294). We are grateful to Davide Elia and Theresa Giannini for providing their unpublished catalogue of dense cores in Vela C. L.M.F. is a Jansky Fellow of the National Radio Astronomy Observatory (NRAO). NRAO is a facility of the National Science Foundation (NSF operated under cooperative agreemen by Associated Universities, Inc. F.P. thanks the European Commission under the Marie Sklodowska-Curie Actions within the H2020 Programme, Grant Agreement number: 658499 PolAME H2020-MSCA-IF-2014. We thank the Columbia Scientific Balloon Facility staff for their outstanding work.

\section{References}

Andersson, B.-G., Lazarian, A., \& Vaillancourt, J. E. 2015, ARA\&A, 53, 501 André, P., Men'shchikov, A., Bontemps, S., et al. 2010, A\&A, 518, L102

André, P., Di Francesco, J., Ward-Thompson, D., et al. 2014, Protostars and Planets VI, 27

Aschenbach, B., Egger, R., \& Trümper, J. 1995, Nature, 373, 587

Baba, D., Nagata, T., Nagayama, T., et al. 2004, ApJ, 614, 818

Bontemps, S., André, P., Könyves, V., et al. 2010, A\&A, 518, L85

Cabral, B., \& Leedom, L. C. 1993, in Special Interest Group on GRAPHics and Interactive Techniques Proceedings, Special Interest Group on GRAPHics and Interactive Techniques Proceedings

Chen, C.-Y., King, P. K., \& Li, Z.-Y. 2016, ApJ, 829, 84

Cox, N. L. J., Arzoumanian, D., André, P., et al. 2016, A\&A, 590, A110

Crutcher, R. M. 2012, ARA\&A, 50, 29

Davis, Jr., L., \& Greenstein, J. L. 1951, ApJ, 114, 206

Fissel, L. M., Ade, P. A. R., Angilè, F. E., et al. 2016, ApJ, 824, 134

Galitzki, N., Ade, P. A. R., Angilè, F. E., et al. 2014, in Ground-based and Airborne Telescopes V, Proc. SPIE, 9145, 91450

Gandilo, N. N., Ade, P. A. R., Angilè, F. E., et al. 2016, ApJ, 824, 84

Giannini, T., Elia, D., Lorenzetti, D., et al. 2012, A\&A, 539, A156

Górski, K. M., Hivon, E., Banday, A. J., et al. 2005, ApJ, 622, 759

Heiles, C., \& Haverkorn, M. 2012, Space Sci. Rev., 166, 293

Hennebelle, P. 2013, A\&A, 556, A153

Hennebelle, P., \& Pérault, M. 2000, A\&A, 359, 1124

Hildebrand, R. H. 1983, QJRAS, 24, 267

Hildebrand, R. H. 1988, QJRAS, 29, 327
Hill, T., Motte, F., Didelon, P., et al. 2011, A\&A, 533, A94

Hill, T., André, P., Arzoumanian, D., et al. 2012, A\&A, 548, L6

Hiltner, W. A. 1949, Science, 109, 165

Inutsuka, S.-I., Inoue, T., Iwasaki, K., \& Hosokawa, T. 2015, A\&A, 580, A49

Kirk, H., Myers, P. C., Bourke, T. L., et al. 2013, ApJ, 766, 115

Könyves, V., André, P., Men'shchikov, A., et al. 2015, A\&A, 584, A91

Kusune, T., Sugitani, K., Nakamura, F., et al. 2016, ApJ, 830, L23

Lazarian, A. 2000, in Cosmic Evolution and Galaxy Formation: Structure, Interactions, and Feedback, eds. J. Franco, L. Terlevich, O. López-Cruz, \& I. Aretxaga, ASP Conf. Ser., 215, 69

Li, H.-B., Fang, M., Henning, T., \& Kainulainen, J. 2013, MNRAS, 436, 3707

Liseau, R., Lorenzetti, D., Nisini, B., Spinoglio, L., \& Moneti, A. 1992, A\&A, 265, 577

Malinen, J., Montier, L., Montillaud, J., et al. 2016, MNRAS, 460, 1934

Matthews, T. G., Ade, P. A. R., Angilè, F. E., et al. 2014, ApJ, 784, 116

May, J., Murphy, D. C., \& Thaddeus, P. 1988, A\&AS, 73, 51

Mestel, L. 1965, QJRAS, 6, 161

Mestel, L., \& Paris, R. B. 1984, A\&A, 136, 98

Mestel, L., \& Spitzer, Jr., L. 1956, MNRAS, 116, 503

Minier, V., Tremblin, P., Hill, T., et al. 2013, A\&A, 550, A50

Montier, L., Plaszczynski, S., Levrier, F., et al. 2015, A\&A, 574, A136

Mouschovias, T. C. 1976, ApJ, 206, 753

Murphy, D. C., \& May, J. 1991, A\&A, 247, 202

Naghizadeh-Khouei, J., \& Clarke, D. 1993, A\&A, 274, 968

Netterfield, C. B., Ade, P. A. R., Bock, J. J., et al. 2009, ApJ, 707, 1824

Ntormousi, E., Dawson, J. R., Hennebelle, P., \& Fierlinger, K. 2017, A\&A, 599, A94

Palmeirim, P., André, P., Kirk, J., et al. 2013, A\&A, 550, A38

Paradis, D., Dobashi, K., Shimoikura, T., et al. 2012, A\&A, 543, A103

Pascale, E., Ade, P. A. R., Bock, J. J., et al. 2008, ApJ, 681, 400

Pascale, E., Ade, P. A. R., Angilè, F. E., et al. 2012, in SPIE Conf. Ser., 8444

Planck Collaboration XI. 2014, A\&A, 571, A11

Planck Collaboration I. 2016, A\&A, 594, A1

Planck Collaboration Int. XIX. 2015, A\&A, 576, A104

Planck Collaboration Int. XXXII. 2016, A\&A, 586, A135

Planck Collaboration Int. XXXV. 2016, A\&A, 586, A138

Prato, L., Rice, E. L., \& Dame, T. M. 2008, Handbook of Stars Forming Regions,

Vol. I: The Northern Sky ASP Monograph Publication, 4, ed. B. Reipurth, 18 Roussel, H. 2013, PASP, 125, 1126

Roy, A., André, P., Palmeirim, P., et al. 2014, A\&A, 562, A138

Santos, F. P., Ade, P. A. R., Angilè, F. E., et al. 2017, ApJ, 837, 161

Serkowski, K. 1958, Acta Astron., 8, 135

Soler, J. D., Hennebelle, P., Martin, P. G., et al. 2013, ApJ, 774, 128

Soler, J. D., Alves, F., Boulanger, F., et al. 2016, A\&A, 596, A93

Sugitani, K., Nakamura, F., Watanabe, M., et al. 2011, ApJ, 734, 63

Walch, S., Girichidis, P., Naab, T., et al. 2015, MNRAS, 454, 238

Yamaguchi, N., Mizuno, N., Saito, H., et al. 1999, PASJ, 51, 775 


\section{Appendix A: The $I_{500} \mathrm{HROs}$}

We construct the histograms of the relative orientations (HROs) using BLASTPol observations of polarization at 250, 350, and $500 \mu \mathrm{m}$ and the gradient of the intensity observed by BLASTPol in the $500 \mu \mathrm{m}$ band, $I_{500}$, using the procedure described in Sect. 4.2. This family of HROs has the advantage of considering observations made with the same instrument, but the obvious disadvantage that $I_{500}$ is merely a proxy for the total gas column density.

The $I_{500}$ HROs, shown in the lower panels of Fig. A.1, are flat in the lowest $I_{500}$ range and peak at $90^{\circ}$ in the intermediate and highest $I_{500}$ ranges across the three BLASTPol wavelength bands. The agreement between observations in different wavebands and the prevalence of the HROs peaking at $90^{\circ}$ is confirmed by the behaviour of $\xi$ as a function of $I_{500}$, shown in Fig. A.2. The figure shows that $\xi<0$ in almost all of the $I_{500}$ bins, although the error bars indicate that this value is only clearly separated from $\xi \approx 0$ in the highest $I_{500}$ bin.

However, the $I_{500} \mathrm{HROs}$ that indicate that $\left\langle\hat{\boldsymbol{B}}_{\perp}\right\rangle$ is mostly perpendicular to $I_{500}$ are not conclusive given that $I_{500}$ is only a proxy to column density. Direct comparison with the trends presented in Planck Collaboration Int. XXXV (2016) would require further evaluation of the total gas column density, something that is readily available through the multi-wavelength observation of this region by Herschel.
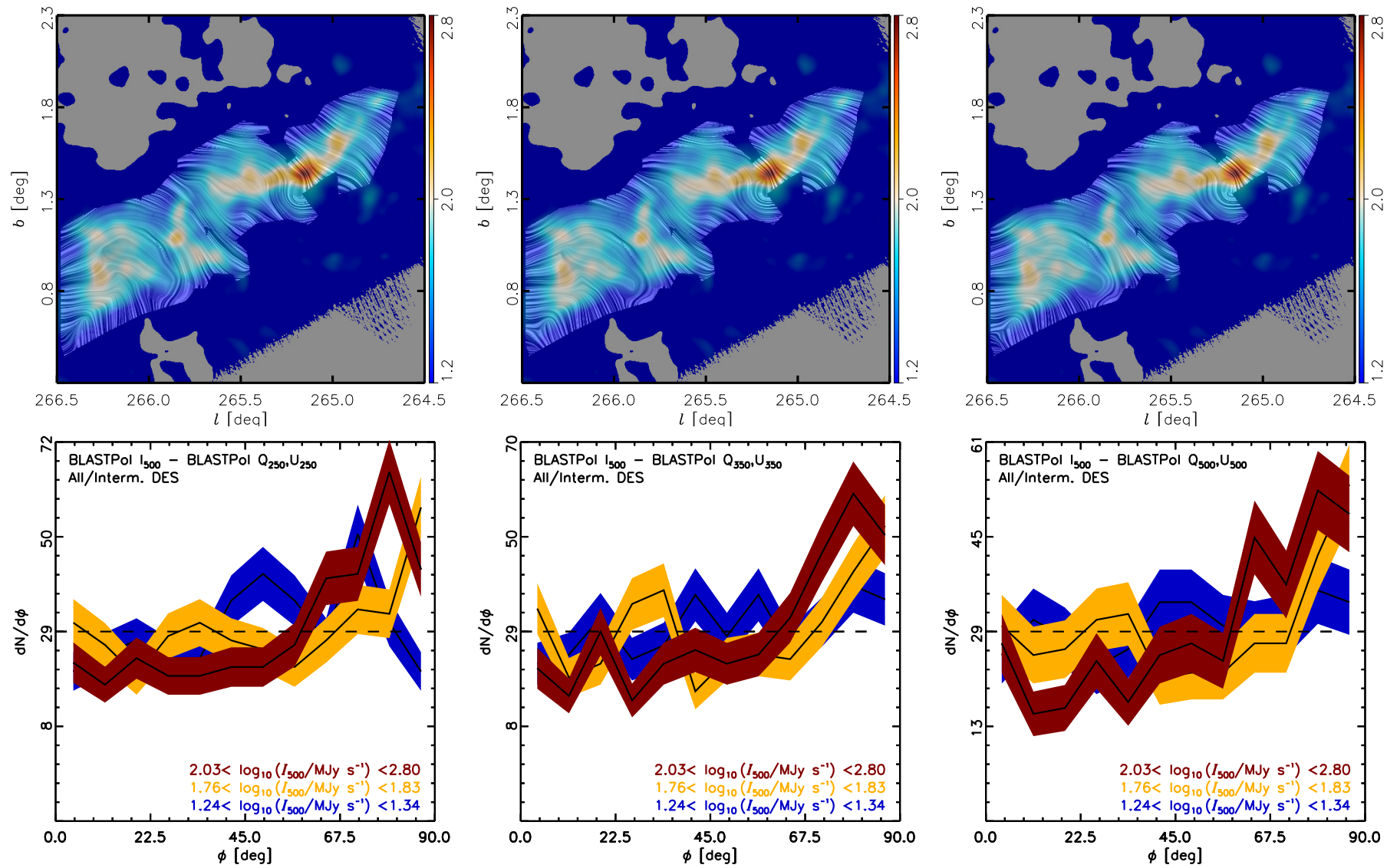

Fig. A.1. Top: magnetic field and total intensity measured by BLASTPol towards two regions in the Vela molecular ridge. The colours represent $I_{500}$, the total intensity observed in the 500- $\mu$ m wavelength-band of BLASTPol. The "drapery" pattern, produced using the line integral convolution method (LIC, Cabral \& Leedom 1993), indicates the orientation of magnetic field lines, orthogonal to the orientation of the submillimetre polarization observed by BLASTPol at 250 (left), 350 (centre), and $500 \mu \mathrm{m}$ (right). Bottom: histogram of the relative orientations (HRO) between the iso- $I_{500}$ contours and the magnetic field orientation inferred from the BLASTPol observations at 250 (left), 350 (centre), and $500 \mu \mathrm{m}$ (right). The figures present the HROs for the lowest, an intermediate, and the highest $I_{500}$ bin (blue, orange, and dark red, respectively). The bins have equal numbers of selected pixels within the $I_{500}$-ranges labelled. The horizontal dashed line corresponds to the average per angle bin of $15^{\circ}$. The widths of the shaded areas for each histogram correspond to the $1 \sigma$ uncertainties related to the histogram binning operation. Histograms peaking at $0^{\circ}$ correspond to $\left\langle\hat{\boldsymbol{B}}_{\perp}\right\rangle$ predominantly aligned with iso- $I_{500}$ contours. Histograms peaking at $90^{\circ}$ correspond to $\left\langle\hat{\boldsymbol{B}}_{\perp}\right\rangle$ predominantly perpendicular to iso- $I_{500}$ contours. 


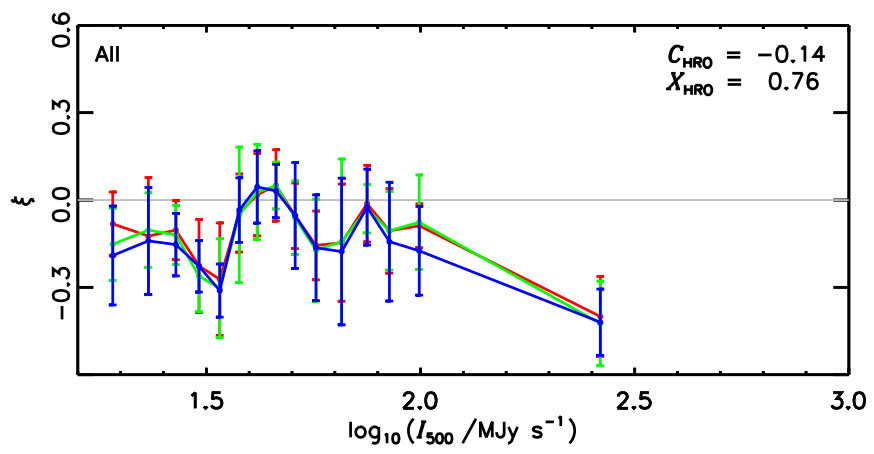

Fig. A.2. Relative orientation parameter $\xi$, defined in Eq. (3), calculated for the different $I_{500}$ bins towards the Vela $\mathrm{C}$ region. The values $\xi>0$ and $\xi<0$ correspond to the magnetic field oriented mostly parallel and perpendicular to the iso- $I_{500}$ or iso- $N_{\mathrm{H}}$ contours, respectively. The grey line corresponds to $\xi=0$, which corresponds to the case there is not a preferred relative orientation.

\section{Appendix B: Diffuse emission subtraction}

The observed polarization degree and orientation angles are the product of the contributions from aligned dust grains in the cloud and in the foreground and background. In order to estimate the contribution of the foreground or background, Fissel et al. (2016) present three different background subtraction methods, one conservative and one more aggressive with respect to diffuse emission subtraction (DES), as described in Sect. 3.1.
Figure B.1 shows the HROs calculated using the BLASTPol $250-\mu \mathrm{m}$ observations, estimated using the three DES methods, towards the South-Ridge sub-region of Vela C. The main differences between the HROs corresponding to the different DES

methods are the amount of jitter, which is similar in the conservative and intermediate DES, but slightly different in the HROs that correspond to the aggressive DES. However, the HROs in Fig. B.1 indicate that the selection of the reference regions for the DES does not significantly affect the relative orientation trends that they describe. This agreement between the HROs corresponding to different DES methods is also found in the other sub-regions of Vela $\mathrm{C}$. The values of the relative orientation parameter, $\xi$, shown in Fig. B.2, also reveal that the selection of DES method does not significantly change the behaviour of $\xi$ as a function of $N_{\mathrm{H}}$.
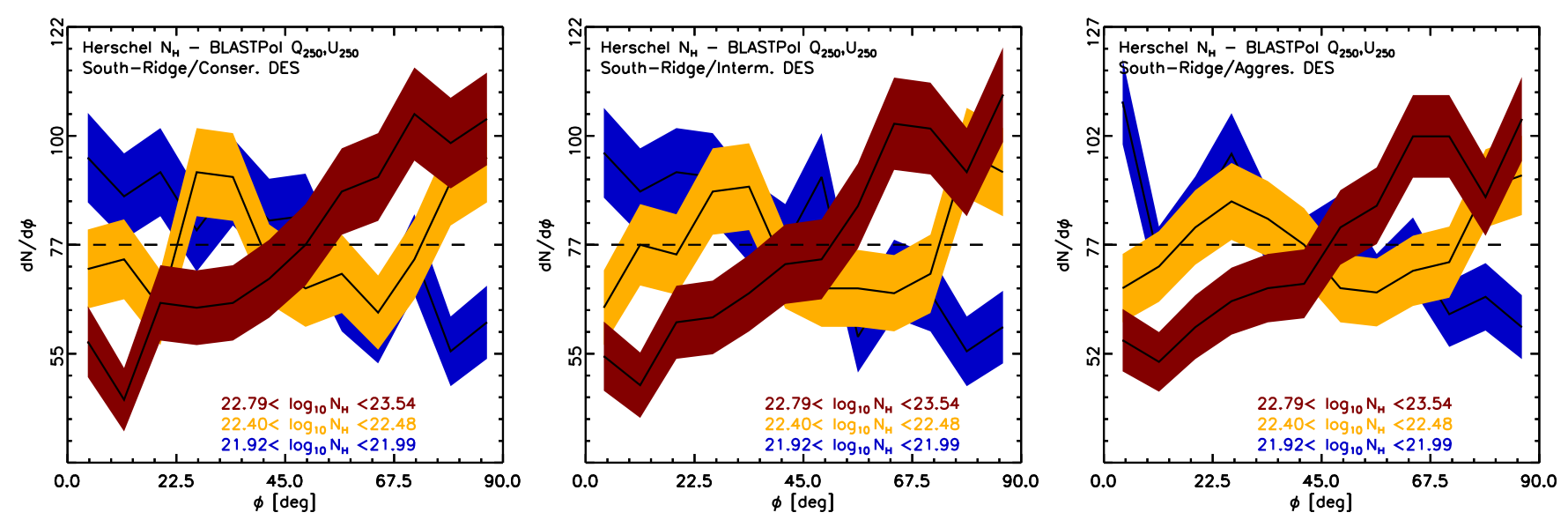

Fig. B.1. Histogram of the relative orientations (HRO) between the iso- $N_{\mathrm{H}}$ contours and the magnetic field orientation inferred from the BLASTPol observations at $250 \mu \mathrm{m}$ obtained with the three diffuse emission subtraction (DES) methods, namely aggressive, intermediate, and conservative, defined in Sect. 3.1. The figures present the HROs for the lowest bin, an intermediate bin, and the highest $N_{\mathrm{H}}$ bin (blue, orange, and dark red, respectively). The bins have equal numbers of selected pixels within the $N_{\mathrm{H}}$ ranges labelled. The horizontal dashed line corresponds to the average. The widths of the shaded areas for each histogram correspond to the $1 \sigma$ uncertainties related to the histogram binning operation. Histograms peaking at $0^{\circ}$ would correspond to $\left\langle\hat{\boldsymbol{B}}_{\perp}\right\rangle$ being predominantly aligned with iso- $N_{\mathrm{H}}$ contours, while histograms peaking at $90^{\circ}$ would correspond to $\left\langle\hat{\boldsymbol{B}}_{\perp}\right\rangle$ predominantly perpendicular to iso- $N_{\mathrm{H}}$ contours. 
J. D. Soler et al.: Relative orientation between $\left\langle\hat{\boldsymbol{B}}_{\perp}\right\rangle$ and $N_{\mathrm{H}}$ structures towards Vela C
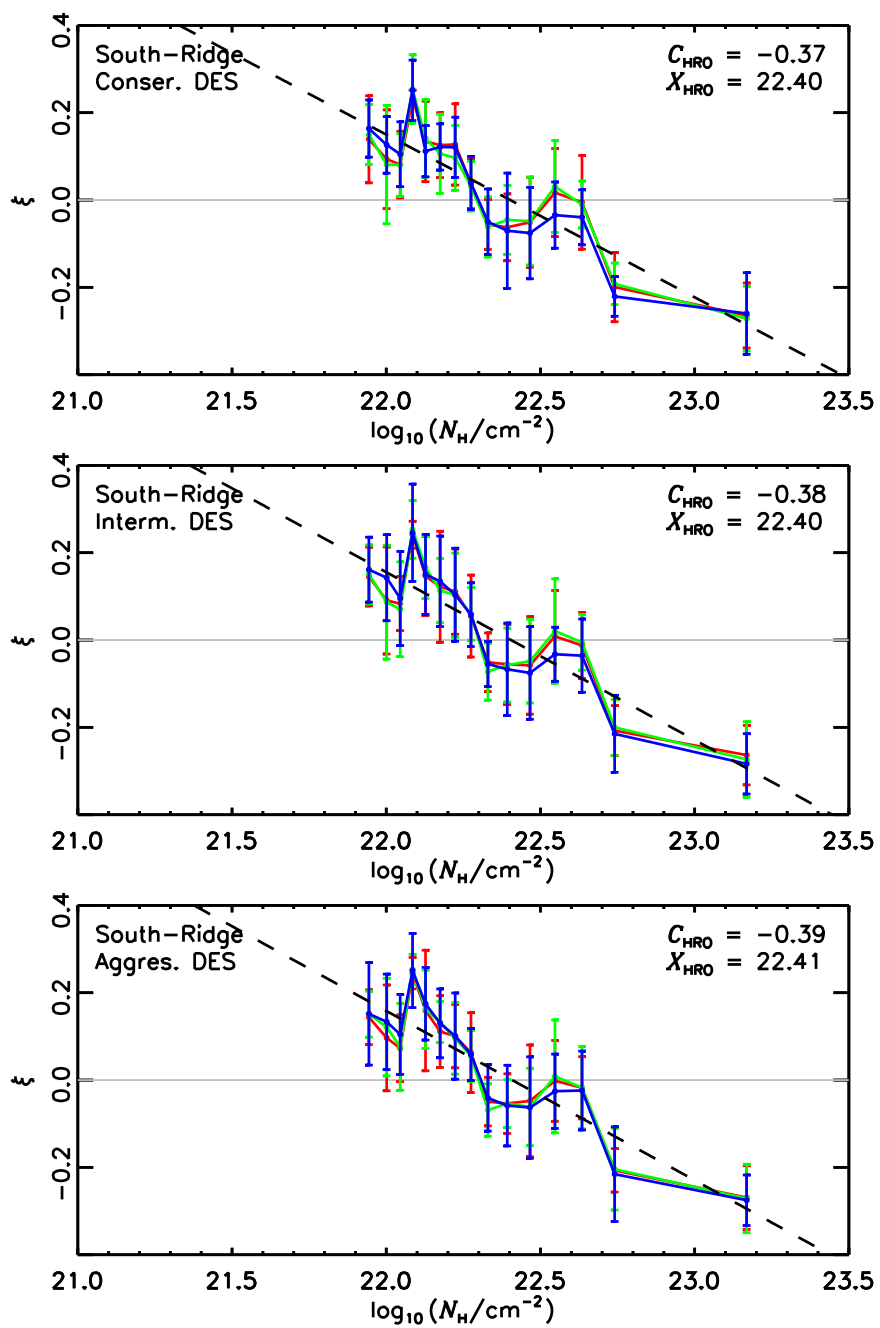

Fig. B.2. Relative orientation parameter $\xi$, defined in Eq. (3), calculated for the different $N_{\mathrm{H}}$ bins towards the two sub-regions of Vela C corresponding to the three diffuse emission subtraction (DES) methods; aggressive, intermediate, and conservative; defined in Sect. 3.1. The values $\xi>0$ and $\xi<0$ correspond to the magnetic field oriented mostly parallel and perpendicular to the iso- $N_{\mathrm{H}}$ contours, respectively. The black dashed line and the values of $C_{\mathrm{HRO}}$ and $X_{\mathrm{HRO}}$ correspond to the linear fit introduced in Eq. (5). The grey line is $\xi=0$, which corresponds to the case where there is no preferred relative orientation. 


\section{Appendix C: The Aquila region}

The Aquila rift is a $5^{\circ}$-long extinction feature above the Galactic plane at Galactic longitudes between $l=30^{\circ}$ and $l=50^{\circ}$ (Prato et al. 2008). In this work, we focus on the $3^{\circ} \times 3^{\circ}$ portion of Aquila rift around $l=28^{\circ}$ and $b=3.5$ observed by Herschel SPIRE at 250, 350, and $500 \mu \mathrm{m}$ and PACS at 70 and $160 \mu \mathrm{m}$ (Bontemps et al. 2010). This region observed by Herschel includes: Serpens South, a young protostellar cluster showing very active recent star formation and embedded in a dense filamentary cloud; W40, a young star cluster associated with the eponymous H II region; and MWC 297, a young $10 M_{\odot}$ star (Könyves et al. 2015, and the references therein).

The whole area covered by Herschel has a total mass of $3.1 \times 10^{4} M_{\odot}$, with a $1.1 \times 10^{4} M_{\odot}$ region associated with W40, and a $4.1 \times 10^{3} M_{\odot}$ region associated with MWC 297 (Bontemps et al. 2010, and references therein). Although this portion of Aquila is less massive than Vela $C$, observations with P-ArTéMiS indicate that the filamentary structures in both regions are similar in column density profiles and mass per unit length (Hill et al. 2012), making it an interesting candidate for comparison using the HRO analysis.

\section{C.1. Observations}

Figure C. 1 shows the $N_{\mathrm{H}}$ and $\left\langle\hat{\boldsymbol{B}}_{\perp}\right\rangle$ maps, and the position of the clumps with $M>3 M_{\odot}$ from the catalogue of dense cores presented in Könyves et al. (2015). At the assumed distance of the Aquila rift, $260 \mathrm{pc}$, these maps are sampling physical scales of 0.12 and $2 \mathrm{pc}$, respectively.

\section{C.1.1. Thermal dust polarization}

We use the Planck $353 \mathrm{GHz}$ Stokes $Q$ and $U$ maps and the associated noise maps made from five independent consecutive sky surveys of the Planck cryogenic mission, which together correspond to the Planck 2015 public data release $^{1}$ (Planck Collaboration I 2016). The whole-sky $353 \mathrm{GHz}$ maps of $Q$ and $U$, their respective variances $\sigma_{\mathrm{Q}}^{2}$ and $\sigma_{\mathrm{U}}^{2}$, and their covariance $\sigma_{\mathrm{QU}}$ are initially at 4.8 resolution in HEALPIX format $^{2}$ (Górski et al. 2005), with a pixelization at $N_{\text {side }}=2048$, which corresponds to an effective pixel size of 1.7. To increase the signal-to-noise ratio $(\mathrm{S} / \mathrm{N})$ of extended emission, we smooth all the maps to $10^{\prime}$ resolution using a Gaussian approximation to the Planck beam and the smoothing procedures for the covariance matrix described in Planck Collaboration Int. XIX (2015).

The maps of the individual regions are projected and resampled onto a Cartesian grid with the gnomonic projection procedure described in Paradis et al. (2012). The present analysis is performed on these projected maps. The selected regions are small enough, and are located at sufficiently low Galactic latitudes, that this projection does not impact significantly on our study.

\section{C.1.2. Column density}

We use the 36 ".5 resolution $N_{\mathrm{H}_{2}}$ column density maps derived from the 70-, 160-, 250-, 350-, and 500- $\mu \mathrm{m}$ Herschel observations, described in Könyves et al. (2015) and publicly available

\footnotetext{
http://pla.esac.esa.int/pla/

2 http://healpix.sf.net
}

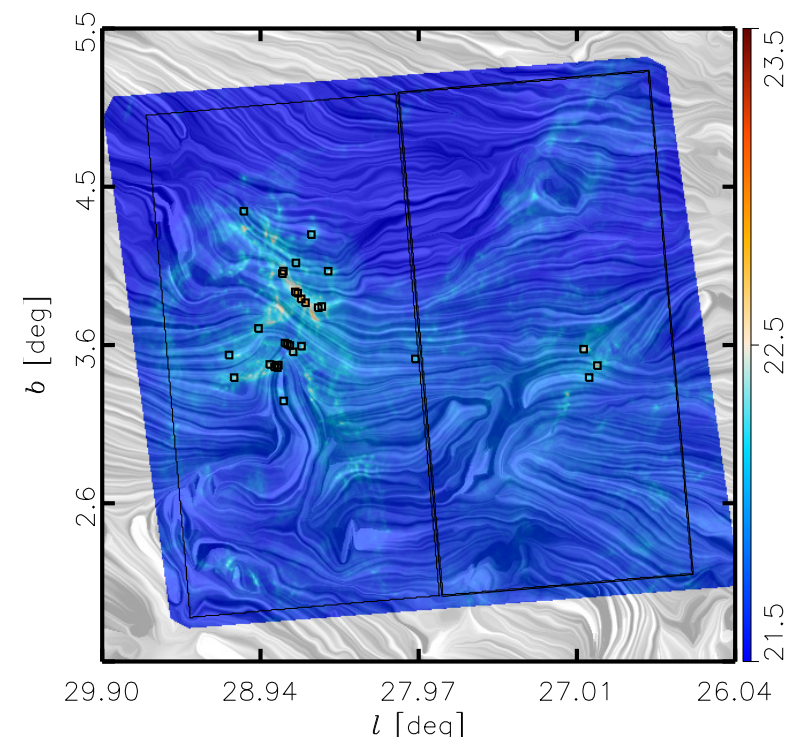

Fig. C.1. Magnetic field and total gas column density measured towards a sub-region of the Aquila rift. The colours represent the total gas column density, $N_{\mathrm{H}}$, inferred from the Herschel observations (Könyves et al. 2015). The "drapery" pattern, produced using the line integral convolution method (LIC, Cabral \& Leedom 1993), indicates the orientation of magnetic field lines, orthogonal to the orientation of the submillimetre polarization observed by Planck at $353 \mathrm{GHz}$. The black squares correspond to the location of the cores with $M>$ $3 M_{\odot}$, pre-stellar and protostellar, from the catalogue presented in Könyves et al. (2015). The black polygons correspond to the two portions where we compare the results of the HRO analysis, which contain W40 (left) and MWC 297 (right), respectively.

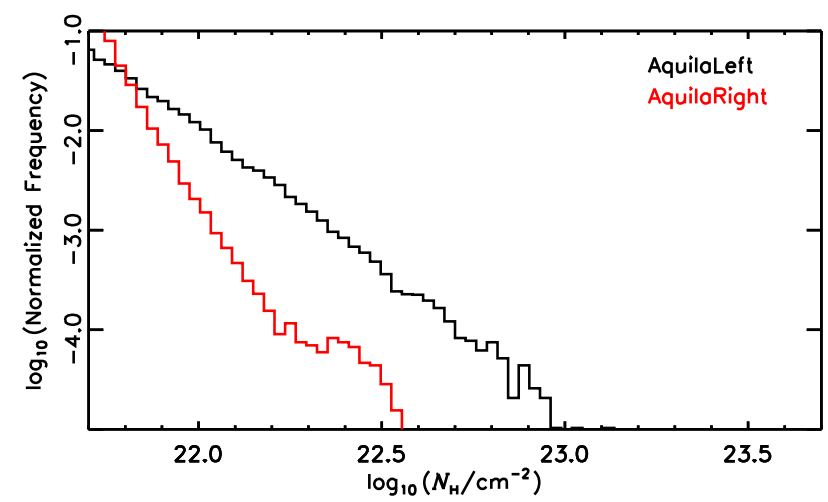

Fig. C.2. Comparison of the total gas column density probability distribution functions (PDFs), inferred from the Herschel observations (Könyves et al. 2015), in the two sub-regions of the Aquila rift presented in Fig. C.1. The $y$-axis is presented in logarithmic scale for the sake of comparison with Fig. 5 of Hill et al. (2011).

in the archive of the Herschel Gould Belt Survey ${ }^{3}$ (HGBS, André et al. 2010) project.

Additionally, we use the catalogue of dense cores identified in the HGBS maps of the Aquila region (Könyves et al. 2015), considering their estimated core mass obtained by assuming the dust opacity law advocated by Roy et al. (2014).

\section{C.2. Analysis}

We apply the analysis described in Sect. 4, using the aforementioned maps and focus on two arbitrary sub-regions with the

\footnotetext{
3 http://www.herschel. fr/cea/gouldbelt
} 

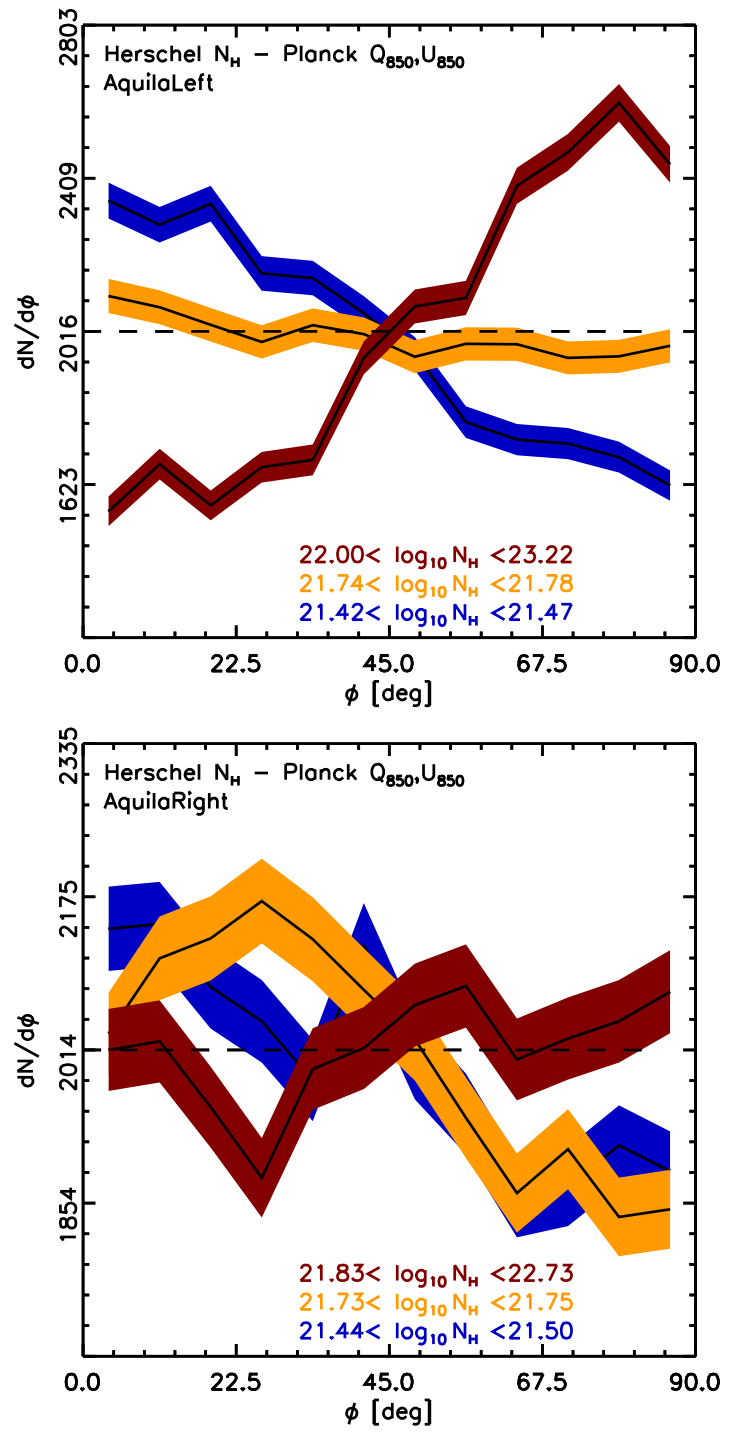

Fig. C.3. Histogram of the relative orientations (HRO) between the iso- $N_{\mathrm{H}_{2}}$ contours and the magnetic field orientation inferred from the Planck $353 \mathrm{GHz}$ observations towards the two regions of the Aquila rift illustrated in Fig. C.1. The figures present the HROs for the lowest, an intermediate, and the highest $N_{\mathrm{H}}$ bin (blue, orange, and dark red, respectively). The bins have equal numbers of selected pixels within the $N_{\mathrm{H}}$-bins ranges labelled. The horizontal dashed line corresponds to the average per angle bin of $15^{\circ}$. The widths of the shaded areas for each histogram correspond to the $1 \sigma$ uncertainties related to the histogram binning operation. Histograms peaking at $0^{\circ}$ correspond to $\left\langle\hat{\boldsymbol{B}}_{\perp}\right\rangle$ predominantly aligned with iso- $N_{\mathrm{H}_{2}}$ contours. Histograms peaking at $90^{\circ}$ correspond to $\left\langle\hat{\boldsymbol{B}}_{\perp}\right\rangle$ predominantly perpendicular to iso- $N_{\mathrm{H}_{2}}$ contours.

same area: one containing the W40 H II region; and the other with MWC 297. Given, that the region around W40 contains the majority of the candidate prestellar and protostellar cores, as clearly show in figure 1 of Könyves et al. (2015), and a shallower high- $N_{\mathrm{H}}$ PDF tail, as shown in Fig. C.2, we aim to evaluate if both characteristics are correlated with the behaviour of the HROs.

Figure C. 3 shows the HROs of the two sub-regions of the Aquila rift. The region containing W40, where most of the candidate prestellar and protostellar cores are located, shows a clear change from the histogram peaking at $0^{\circ}$ in the lowest $N_{\mathrm{H}_{2}}{ }^{-}$ bin (indicating $\left\langle\hat{\boldsymbol{B}}_{\perp}\right\rangle$ mostly parallel to the $N_{\mathrm{H}}$ structures) to the
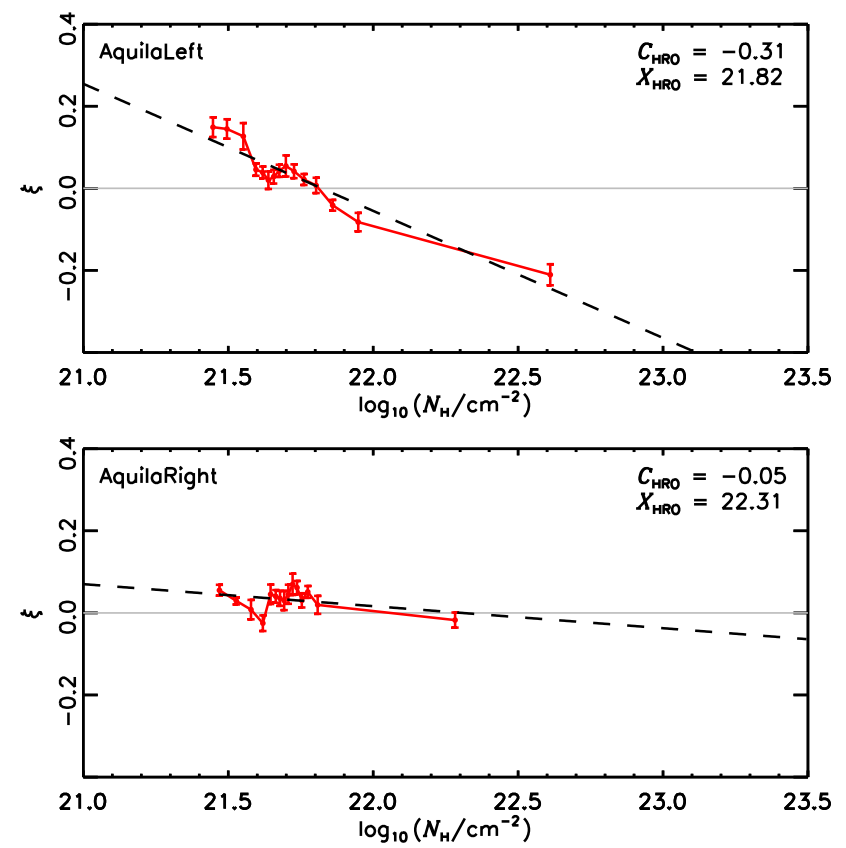

Fig. C.4. Relative orientation parameter $\xi$, defined in Eq. (3), calculated for the different $N_{\mathrm{H}}$ bins towards the two portions of the Aquila rift illustrated in Fig. C.1. The values $\xi>0$ and $\xi<0$ correspond to the magnetic field oriented mostly parallel and perpendicular to the iso- $N_{\mathrm{H}}$ contours, respectively. The black dashed line and the values of $C_{\mathrm{HRO}}$ and $X_{\mathrm{HRO}}$ correspond to the linear fit introduced in Eq. (5). The grey line is $\xi=0$, which corresponds to the case where there is no preferred relative orientation.

histogram peaking at $90^{\circ}$ in the highest $N_{\mathrm{H}^{-}}$bin (indicating $\left\langle\hat{\boldsymbol{B}}_{\perp}\right\rangle$ mostly perpendicular to the $N_{\mathrm{H}}$ structures). In contrast, the region containing MWC 297, shows histograms peaking at $0^{\circ}$ in the lowest and intermediate $N_{\mathrm{H}}$-bins, while the $\mathrm{HRO}$ in the highest $N_{\mathrm{H}}$-bin has a lot of jitter, but is consistent with no preferential relative orientation between $N_{\mathrm{H}}$ and $\left\langle\hat{\boldsymbol{B}}_{\perp}\right\rangle$. However, the region containing MWC 297 has a lower maximum $N_{\mathrm{H}}$ value, and thus, a more complete evaluation of the changes in the HRO should be made in terms of $\xi$.

Figure C. 4 shows the relative orientation parameter, $\xi$ as defined in Eq. (3), as a function of $N_{\mathrm{H}}$ in both sub-regions of the Aquila rift. The values of $\xi$ and $C_{\mathrm{HRO}}$ indicate that the region containing $\mathrm{W} 40$ has a clear change in the relative orientation between $N_{\mathrm{H}}$ and $\left\langle\hat{\boldsymbol{B}}_{\perp}\right\rangle$, from mostly parallel to mostly perpendicular with increasing $N_{\mathrm{H}}$. In the region containing MWC 297, $C_{\mathrm{HRO}}$ is also closer to zero and $\xi$ is closer to zero, suggesting a small change in relative orientation between $N_{\mathrm{H}}$ and $\left\langle\hat{\boldsymbol{B}}_{\perp}\right\rangle$ from mostly parallel to mostly perpendicular with increasing $N_{\mathrm{H}}$ but with values of $\xi$ suggesting no preferential relative orientation.

The behaviour of $\xi$ as a function of $N_{\mathrm{H}}$ is in clear agreement with that reported for a much larger portion of the Aquila rift in Planck Collaboration Int. XXXV (2016); however, the comparison with the higher-resolution Herschel map shows that different portions of the cloud have different degrees of change in relative orientation between $N_{\mathrm{H}}$ and $\left\langle\hat{\boldsymbol{B}}_{\perp}\right\rangle$. In the sub-region of the Aquila rift presented in this work, we found a sharper transition between $N_{\mathrm{H}}$ being preferentially parallel or having no preferred relative orientation with respect to $\left\langle\hat{\boldsymbol{B}}_{\perp}\right\rangle$ at the low $N_{\mathrm{H}}$, to being preferentially perpendicular at the highest $N_{\mathrm{H}}$, in the portion of the cloud with the greatest values of $N_{\mathrm{H}}$, the flattest high-column density tail of the PDF, and the largest number of prestellar and protostellar cores. 